\title{
FIRST OBSERVATIONS WITH THE EFFELSBERG RADIO TELESCOPE AT 7-MM WAVELENGTH. II. A SURVEY OF STARS IN THE VIBRATIONALLY EXCITED $J=1-0$ SiO LINES
}

\section{J. H. SPENCER}

E. O. Hulburt Center for Space Research, Naval Research Laboratory, Washington, D.C. 20375

\author{
A. WINNBERG \\ Max-Planck-Institut für Radioastronomie, Auf dem Hügel 69, D-5300 Bonn 1, West Germany \\ F. M. OLNON \\ Sterrewacht-Huygens Laboratorium, Wassenaarsweg 78, Leiden 2405, The Netherlands \\ P. R. SCHWARTZ \\ E. O. Hulburt Center for Space Research, Naval Research Laboratory, Washington, D.C. 20375 \\ H. E. MatThews \\ Herzberg Institute of Astrophysics, National Research Council, Ottawa, Ontario K1A 0R6, Canada

\section{DOWNES} \\ Institut de Radio Astronomie Millimetrique, B.P. 391, 38017 Grenoble, France \\ Received 14 October 1980; revised 24 November 1980
}

\begin{abstract}
High-resolution and high-sensitivity spectra are presented for $46 \mathrm{SiO}$ masers in the $J=1-0$ transitions of the $v=1, v=2$, and $v=3$ excited states. The emission is a complex blend of weak maser lines. Our sample is statistically complete to a distance of approximately $300 \mathrm{pc}$, but is sensitivity limited. The luminosity functions are the same for $v=1$ and $v=2$ regardless of their shape, but the $v=1$ line is, on the average, 1.4 times stronger than the $v=2$ line in the same star. A luminosity function corrected for incompleteness has been obtained using a new method. The SiO luminosity is not correlated with other stellar properties, which indicates that local conditions in the stellar atmospheres determine the maser intensity.
\end{abstract}

\section{INTRODUCTION}

$\mathrm{SiO}$ masers provide important information on the physical conditions in circumstellar shells and the mechanisms of mass loss. Visual and infrared observations limit possible models of these stellar masers more than for masers in regions of star formation. The $\mathrm{SiO}$ maser remains unique in that it is found only in late-type stars, with the possible exception of the source IRc 2 in Orion (Genzel et al. 1980). Because of their large distance and complexity, late-type stars are not as well understood as other types of stars. Many of their known properties are derived from statistical techniques. With the number of $\mathrm{SiO}$ masers now known, it is possible to do statistical studies to derive some of the physical properties of these stars.

A high-sensitivity survey of 7-mm SiO lines of a large number of stars, conducted within a short time $(\sim 1$ month), can be used to test for correlations between the maser intensity and parameters of the stellar envelopes. Nearly simultaneous observations at different vibrational states limit uncertainties due to maser variations in these maser model tests.

In the spring of 1979 the $100-\mathrm{m}$ radio telescope in Effelsberg became available for 7-mm observations and a survey of this type was attempted. In this paper we report the results of the observations of the vibrationally excited $J=1-0 \mathrm{SiO}$ lines. A separate paper on the ground-state $v=0$ observations of these stars is in preparation.

\section{OBSERVATIONS}

The receiver and telescope characteristics have been described by Altenhoff $e t$ al. (1980, hereafter referred to as Paper I). The observations were taken in a totalpower on-off mode with total on-source integration times of 5 to $10 \mathrm{~min}$. Individual on-source components of this integration time were between 1 and $3 \mathrm{~min}$. The $E$ vector of polarization was vertical, producing a rotation of the position angle of the $E$ vector as the source was tracked across the sky. 
Most of the spectra reported here were taken with an autocorrelator, with spectral resolutions of 62.5 or 31.3 $\mathrm{kHz}\left(\sim 0.44\right.$ or $\left.\sim 0.22 \mathrm{~km} \mathrm{~s}^{-1}\right)$ and a total bandwidth of 10 or $5 \mathrm{MHz}$, respectively. The $v=1$ and $v=2$ lines were observed simultaneously in the two parts of the autocorrelator with 192 channels for each. We also used a filter spectrometer consisting of 128 filters with widths of $312.5 \mathrm{kHz}\left(\sim 2.2 \mathrm{~km} \mathrm{~s}^{-1}\right)$. These filters were shared between either $v=1$ and $v=2$, or $v=0$ and $v=3$, to give an effective filter spectrometer of 50 to 65 filters with a velocity range of 105 to $140 \mathrm{~km} \mathrm{~s}^{-1}$. In addition to the spectra presented in this paper, we observed several of the strong $\mathrm{SiO}$ masers with the filter spectrometer.

Because the number of known $\mathrm{SiO}$ masers was not statistically large, the present observations included many stars that were not known to have $\mathrm{SiO}$ maser emission in an attempt to increase the number of known masers.

The selection criteria for the search for new $\mathrm{SiO}$ masers included:

(1) Mira variables from Keenan et al. (1974) with $m-M<9.8^{\mathrm{m}}$ (corresponding to $D<900 \mathrm{pc}$ ) and with $\delta>-10^{\circ}$.

(2) IRC sources (Neugebauer and Leighton 1969) with $K \leq 2$ and $I-K \geq 5$ and with $\delta>-10^{\circ}$.

(3) Stars known to have associated masers (either $\mathrm{OH}, \mathrm{H}_{2} \mathrm{O}$, or $\mathrm{SiO}$ ) (cf. Kleinmann et al. 1978 or Engels 1979).

(4) CRL sources with photometry and good positions (Low et al. 1976).

(5) Peculiar stars such as P Cyg or MWC 349.

We also included some pre-main-sequence stars like $\mathrm{T}$ Tauri stars and M supergiants like $\alpha$ Ori that do not fit any of these criteria exactly. The results of observations on early-type stars and regions of star formation are discussed in Genzel et al. (1980). Until the mechanisms of masers associated with stars are better understood, these objects will continue to be included in the observing lists, thus making them statistically incomplete and contaminated.

Except for the unusual object IRC+10216, the list of stars to search for $v=3$ emission was composed from the list of known $\mathrm{SiO} v=1$ and $v=2$ emission stars. In the case of IRC+10216 ( $=\mathrm{CW}$ Leo) the $v=3$ search was conducted for technical reasons as part of the principal $v=0$ search. The amount of time spent searching a star for $v=3$ was determined as much by observing pressure as by $a$ priori expectations.

\section{RESULTS}

The results are listed in Tables I-III. Stars with a positive detection in either the $v=1$ or $v=2$ transition are to be found in Table I. The spectra of these stars are presented in Figs. 1-8. The name of the star is given in column 1, with the IRC number in column 2 . The distances (column 3 of Table I) are derived from the mass, luminosity, period, and spectral type at maximum (Cahn and Wyatt 1978; Cahn 1980). Stars that are not Miras or do not fit the Cahn and Wyatt criteria were estimated by other techniques as noted. Some distances were calculated from the relation between period and absolute visual magnitude $M_{v}$ at mean maximum as given by Foy et al. (1975). The interstellar absorption $A_{v}$ was estimated from an assumed extinction law:

$$
A_{v}=0.14 \csc b[1-\exp (-0.01 d \sin b)],
$$

where $d$ is the distance in parsecs and $b$ is the galactic latitude. Further, stars whose distances were calculated in this manner were rarely used in the detailed analysis of Sec. IV. Distances for supergiants and some infrared stars are from Hyland et al. (1972), except for VY CMa and S Per. The Herbig (1969) distance of $1500 \mathrm{pc}$ was adopted for VY CMa, while S Per was assumed to be associated with the $h$ and $\chi$ Per clusters (Kukarkin et al. 1969-1976). The phase of the optical light cycle is listed in column 4 of Table I. Columns 5 and 6 list the strongest flux density at $v=1$ and $v=2$, while columns 7 and 8 list the radial velocities at which each occurs. The position angle of the $E$ vector of polarization for the observation is given in column 9. Pairs of numbers refer to separate $v=1$ (first) and $v=2$ (second) measurements. The integrated flux of the entire velocity range after a linear baseline was removed is given in columns 10 and 11 . These columns and the distance from column 3 lead to the luminosities given in columns 12 and 13 . The first published $\mathrm{SiO}$ maser reference for each star is listed in column 14 and the notes.

Negative results are listed in Table II, along with the integration time and spectrometer. $\mathrm{AK} / 10$ and $\mathrm{AK} / 5$ refer to the autocorrelator at bandwidths of 10 and 5 $\mathrm{MHz}$; FISPE refers to the filter spectrometer. The $3 \sigma$ noise limits are given for each of $v=1$ and $v=2$.

We searched 13 of the stars in Table I for $v=3$ emission, finding it in the direction only of VX Sgr, as previously reported (Scalise and Lépine 1978), and VY $\mathrm{CMa}$. The peak flux density and radial velocity of our detections are presented in Table III, as well as the $3 \sigma$ noise limits in other stars.

\section{DISCUSSION}

The high-resolution spectra of Figs. 1-8 represent the best basis for the study of $\mathrm{SiO}$ masers yet available. Forty-six of the approximately 75 stars known to have $\mathrm{SiO}$ masers are included in a homogeneous highresolution study. This sample is large enough that the general properties of $\mathrm{SiO}$ maser emission can be studied without referring to the peculiarities of a single source or a small number of sources.

Of the 46 detected stars, 12 are identified in Table I as new detections at $7 \mathrm{~mm}$ : 11 masers associated with classical Mira variables and $\mathrm{OH} 26.5+0.6$, the first Type II OH/IR source detected in any $\mathrm{SiO}$ line $(\mathrm{OH}$ detected at 1612,1665 , and 1667 by Andersson et al. $1974, \mathrm{H}_{2} \mathrm{O}$ detected by Olnon et al. 1980). RX Boo was previously 
TABLE I. Positive results.

\begin{tabular}{|c|c|c|c|c|c|c|c|c|c|c|c|c|c|c|c|}
\hline \multirow[b]{2}{*}{$\begin{array}{c}\text { Star } \\
(1)\end{array}$} & \multirow[b]{2}{*}{$\begin{array}{l}\text { IRC No. } \\
\quad(2)\end{array}$} & \multirow{2}{*}{$\begin{array}{l}\text { Dist. } \\
\text { (pc) } \\
(3)\end{array}$} & \multirow[b]{2}{*}{$\begin{array}{c}\text { Phase } \\
\text { (4) }\end{array}$} & \multicolumn{4}{|c|}{ Strongest feature } & \multirow{2}{*}{$\begin{array}{l}\text { Pol. } \\
\text { angle } \\
\text { (deg) } \\
(9)\end{array}$} & \multicolumn{4}{|c|}{$\begin{array}{c}\text { Integrated flux } \\
\left(\times 10^{-20} \mathrm{~W} \mathrm{~m}^{-2}\right)\end{array}$} & \multicolumn{2}{|c|}{$\begin{array}{l}\text { Log luminosity } \\
\text { (W) }\end{array}$} & \multirow[b]{2}{*}{$\begin{array}{l}\text { Ref } \\
(14)\end{array}$} \\
\hline & & & & $\begin{array}{c}v=1 \\
(5)\end{array}$ & $\begin{array}{c}v=2 \\
(6)\end{array}$ & $\begin{array}{c}v=1 \\
(7)\end{array}$ & $\begin{array}{c}v=2 \\
(8)\end{array}$ & & $\begin{array}{c}v=1 \\
(10)\end{array}$ & & $\begin{array}{c}v= \\
(11)\end{array}$ & & $\begin{array}{c}\overline{v=1} \\
(12)\end{array}$ & $\begin{array}{c}v=2 \\
(13)\end{array}$ & \\
\hline $\mathrm{R}$ Aqr & -20642 & 233 & $0.16^{\mathrm{a}}$ & 270 & 95 & -26 & -26 & -14 & $100 \pm$ & 20 & $40 \pm$ & 10 & 20.81 & 20.41 & 8 \\
\hline W Aqr & +00489 & $406^{\mathrm{b}}$ & $0.90^{\mathrm{a}}$ & 20 & 23 & +1 & +2 & -13 & $15 \pm$ & 3 & $7 \pm$ & 5 & 20.46 & 20.15 & 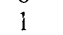 \\
\hline $\mathrm{R} \mathrm{Aql}$ & +10406 & 203 & $0.48^{\mathrm{a}}$ & 17 & 20 & +46 & +46 & -11 & $11 \pm$ & 5 & $2 \pm$ & 3 & 19.73 & 18.99 & 2 \\
\hline RR Aql & +00458 & 446 & $0.71^{\mathrm{a}}$ & 91 & 56 & 29 & 29 & -2 & $22 \pm$ & 5 & $10 \pm$ & 4 & 20.72 & 20.37 & 2 \\
\hline RT Aql & +10433 & 518 & $0.94^{\mathrm{a}}$ & 37 & 14 & -28 & -26 & 9 & $12 \pm$ & 4 & $5 \pm$ & 3 & 20.58 & 20.20 & 7 \\
\hline GY Aql & -10524 & $582^{\mathrm{b}}$ & & 69 & 98 & +30 & +35 & +14 & $41 \pm$ & 8 & $50 \pm$ & 10 & 21.23 & 21.30 & 5 \\
\hline $\mathrm{V} 1300 \mathrm{Aql}$ & -10529 & $620^{\mathrm{d}}$ & $0.6^{\mathrm{f}}$ & 32 & 40 & -19 & -20 & -14 & $7 \pm$ & 6 & $14 \pm$ & 6 & 20.51 & 20.81 & 6 \\
\hline U Ari & +10040 & 531 & $0.21^{\mathrm{a}}$ & 34 & 34 & -57 & -57 & -4 & $7 \pm$ & 2 & $8 \pm$ & 4 & 20.37 & 20.43 & 1 \\
\hline $\mathrm{RX}$ Boo & +30257 & $563^{b}$ & 0.21 & 18 & 30 & -1 & +4 & -19 & $9 \pm$ & 2 & $13 \pm$ & 3 & 20.53 & 20.69 & 3 \\
\hline $\mathrm{R} C \mathrm{nc}$ & +10185 & 296 & 0.44 & 33 & 27 & 16 & 15 & 32 & $22 \pm$ & 4 & $7 \pm$ & 6 & 20.36 & 19.86 & 4,6 \\
\hline VY CMa & -30087 & $1500^{c}$ & & 600 & 580 & 13 & 13 & -15 & $940 \pm 1$ & 190 & $390 \pm$ & 80 & 23.40 & 23.02 & 2 \\
\hline R Cas & +50484 & 182 & $0.9^{\mathrm{a}}$ & 680 & 270 & 26 & 28 & 60 & $360 \pm$ & 70 & $180 \pm$ & 40 & 21.15 & 20.85 & 2 \\
\hline T Cas & +60009 & 239 & $0.7^{\mathrm{a}}$ & 30 & $\leq 20$ & +1 & - & 26 & $3 \pm$ & 3 & - & & 19.30 & - & 1 \\
\hline Y Cas & +60001 & 506 & $0.64^{\mathrm{a}}$ & 15 & 21 & -17 & -17 & -45 & $10 \pm$ & 5 & $7 \pm$ & 4 & 20.48 & 20.32 & 7 \\
\hline Z Cas & +60418 & 1202 & $0.21^{\mathrm{a}}$ & 19 & 20 & -28 & -28 & -45 & $10 \pm$ & 4 & $12 \pm$ & 4 & 21.23 & 21.32 & 1 \\
\hline Т Сep & +70168 & 188 & $0.6^{\mathrm{a}}$ & 64 & $\leq 24$ & -3 & - & 54 & $31 \pm$ & 5 & - & & 20.11 & - & 4,6 \\
\hline$o$ Cet & +00030 & 90 & 0 & 144 & 172 & 48 & 48 & 7 & $33 \pm$ & 6 & $33 \pm$ & 7 & 19.51 & 19.51 & 2 \\
\hline S CrB & +30272 & 366 & $0.35^{\mathrm{a}}$ & 148 & 109 & -1 & -1 & 20,33 & $27 \pm$ & 3 & $27 \pm$ & 5 & 20.65 & 20.65 & 2 \\
\hline UX Cyg & +30464 & 1180 & $0.37^{\mathrm{a}}$ & 29 & 24 & 3 & 3 & -47 & $16 \pm$ & 7 & $13 \pm$ & 4 & 21.43 & 21.34 & 7 \\
\hline$\chi$ Cyg & +30395 & $128^{\mathrm{b}}$ & $0.70^{\mathrm{a}}$ & 32 & $\leq 12$ & 9 & - & -15 & $9 \pm$ & 3 & - & & 19.24 & - & 2 \\
\hline NML Cyg & + & $500^{\mathrm{d}}$ & & 350 & 220 & 4 & 4 & 42,5 & $140 \pm$ & 20 & $82 \pm$ & 10 & 21.62 & 21.38 & 3 \\
\hline U Her & 98 & 316 & $0.66^{\mathrm{a}}$ & 24 & 24 & -16 & -18 & -21 & $10 \pm$ & 7 & $7 \pm$ & 6 & 20.08 & 19.92 & 2 \\
\hline RU Her & t & 532 & $0.83^{\mathrm{a}}$ & 61 & 49 & -11 & -11 & -27 & $29 \pm$ & 6 & $26 \pm$ & 6 & 0 & 20.94 & 6 \\
\hline R Hya & -20254 & 131 & $0.2^{\mathrm{a}}$ & 77 & 110 & -10 & -11 & 7 & $33 \pm$. & $\cdot 10$ & $40 \pm$ & 10 & 19.83 & 19.91 & 2 \\
\hline W Hya & -30207 & $288^{\mathrm{b}}$ & & 1180 & 680 & 41 & 41 & 5 & $610 \pm 1$ & 120 & $280 \pm$ & 60 & 21 & 21.45 & 2 \\
\hline X Hya & & 436 & $0.6^{\mathrm{a}}$ & 16 & 18 & 26 & 27 & -17 & $13 \pm$ & 7 & $18 \pm$ & 6 & 20.46 & 20.61 & 1 \\
\hline R Leo & +1021 & $244^{\mathrm{b}}$ & $0.4^{\mathrm{a}}$ & 330 & 270 & 4 & 1 & -20 & $200 \pm$ & 40 & $150 \pm$ & 30 & & 21 & 2 \\
\hline $\mathrm{R}$ LMi & +30215 & 300 & $0.16^{\mathrm{a}}$ & 235 & 190 & 1 & 1 & -47 & $90 \pm$ & 20 & $60 \pm$ & 10 & 20.99 & 20.81 & 4 \\
\hline GX Mon & & $1367^{b}$ & $0.19^{\mathrm{a}}$ & 14 & 15 & -7 & -10 & -20 & $5 \pm$ & 5 & $6 \pm$ & 6 & 21.04 & 21.03 & 1 \\
\hline V1111 Oph & +10365 & $500^{d}$ & & 57 & 48 & -30 & -32 & -36 & $34 \pm$ & 8 & $26 \pm$ & 8 & 21.00 & 20.89 & 5 \\
\hline S Ori & & 402 & $0.13^{a}$ & 59 & 51 & 15 & 13 & -21 & $34 \pm$ & 8 & $13 \pm$ & 7 & 2 & 20.40 & 1 \\
\hline U Ori & & 267 & 0.6 & 34 & 22 & -33 & -31 & - & $15 \pm$ & 6 & $7 \pm$ & 3 & & 19.77 & 3 \\
\hline R Peg & +1052 & 411 & $0.14^{\mathrm{a}}$ & 102 & 76 & 23 & 23 & -26 & $70 \pm$ & 10 & $39 \pm$ & 7 & 21.15 & 20.90 & 4 \\
\hline W Peg & & 307 & $0.56^{\mathrm{a}}$ & 18 & 27 & -17 & -17 & -45 & $6 \pm$ & 3 & $8 \pm$ & 3 & 19.83 & 19.95 & 1 \\
\hline S Per & +60088 & $2250^{\mathrm{e}}$ & & 45 & 36 & -43 & -43 & -31 & $30 \pm$ & 6 & $21 \pm$ & 4 & 22.26 & 22.10 & 3 \\
\hline WX Psc & +10011 & $510^{\mathrm{d}}$ & 0.6 & 70 & 75 & 8 & 8 & -33 & $19 \pm$ & 5 & $24 \pm$ & 5 & 20.77 & 20.87 & 6 \\
\hline Z Pup & -20133 & 853 & $0.93^{\mathrm{a}}$ & 55 & $\leq 30$ & +1 & - & -2 & $18 \pm$ & 8 & - & & 21.20 & - & 7 \\
\hline $\mathrm{VX} \mathrm{Sgr}$ & -20431 & $500^{d}$ & & 1590 & 850 & 4 & 3 & 2 & $1370 \pm 2$ & 270 & $710 \pm$ & 140 & 22.61 & 22.32 & 2 \\
\hline R Ser & +20285 & 382 & $0.69^{\mathrm{a}}$ & 55 & 53 & 29 & 29 & 34 & $12 \pm$ & 3 & $11 \pm$ & 3 & 20.32 & 20.28 & 1 \\
\hline & +10290 & $767^{b}$ & $0.26^{\mathrm{a}}$ & 29 & $\leq 18$ & 29 & - & 35 & $10 \pm$ & 8 & 二 & & 20.85 & - & 1 \\
\hline WX Ser & +20281 & $970^{d}$ & $0.05^{\mathrm{a}}$ & 61 & 24 & 7 & 9 & 36 & $33 \pm$ & 9 & $14 \pm$ & 5 & 21.57 & 21.20 & 2 \\
\hline $\mathrm{R}$ Tau & +10060 & $421^{\mathrm{b}}$ & $0.03^{\mathrm{a}}$ & 32 & 28 & 13 & 14 & 16 & $9 \pm$ & 5 & $13 \pm$ & 5 & & 20.43 & 8 \\
\hline IK Tau & +10050 & 284 & $0.1^{\mathrm{a}}$ & 125 & 100 & 39 & 39 & 31 & $90 \pm$ & 20 & $80 \pm$ & 20 & 20.94 & 20.89 & 2 \\
\hline RS Vir & +00243 & $642^{\mathrm{b}}$ & $0.1^{\mathrm{a}}$ & 76 & 34 & -14 & -15 & -23 & $17 \pm$ & 6 & $9 \pm$ & 5 & 20.92 & 20.64 & 1 \\
\hline RT Vir & +10262 & $1348^{b}$ & & 25 & $\leq 22$ & 13 & - & -19 & $18 \pm$ & 5 & 二 & & 21.59 & - & 4 \\
\hline OH $26.5+0.6$ & & - & - & 7 & 8 & 29 & 28 & -13 & $1 \pm$ & 1 & $5 \pm$ & 2 & - & - & 1 \\
\hline
\end{tabular}

${ }^{a}$ Included in statistical analysis.

b Distance determined from absolute magnitude from Foy et al. 1975 (see text).

c Distance from Herbig (1969).

d Distance from Hyland et al. (1972).

e Distance of $h$ and $\chi$ Perseus clusters Allen (1973).

f Phase from Harvey et al. (1974).

1. This paper.

References

2. Snyder and Buhl (1975)

3. Kaifu, Buhl, and Snyder (1975).

4. Spencer et al. (1977).

5. Balister et al. (1977).

6. Blair and Dickinson (1977).

7. Dickinson et al. (1978b).

8. Lépine, Le Squeren, and Scalise (1978).

known as an $86-\mathrm{GHz} \mathrm{SiO}$ emitter (Kaifu, Buhl, and Snyder 1975).

Variation of $\mathrm{SiO}$ maser intensity similar to that reported for $\mathrm{OH}$ and $\mathrm{H}_{2} \mathrm{O}$ masers has been reported (Spencer and Schwartz 1975; Hjalmarson and Olofsson 1979). The data are still too sparse for a meaningful analysis and, thus, we have not corrected our data for periodic variation of maser strength.

\section{a) Velocities and Line Profiles}

$\mathrm{SiO}$ maser emission is restricted to a small velocity 
TABLE II. Negative results $(3 \sigma)$.

\begin{tabular}{|c|c|c|c|c|c|c|c|c|c|}
\hline \multirow[b]{2}{*}{ Star } & \multirow{2}{*}{$\begin{array}{c}\text { Integration } \\
\text { time (s) }\end{array}$} & \multirow{2}{*}{$\begin{array}{c}\text { Spec- } \\
\text { trometer }\end{array}$} & \multicolumn{2}{|c|}{$\begin{array}{l}\text { Flux density } \\
\text { limit (Jy) }\end{array}$} & \multirow[b]{2}{*}{ Star } & \multirow{2}{*}{$\begin{array}{l}\text { Integration } \\
\text { time }(s)\end{array}$} & \multirow{2}{*}{$\begin{array}{l}\text { Spec- } \\
\text { trometer }\end{array}$} & \multicolumn{2}{|c|}{$\begin{array}{c}\text { Flux density } \\
\text { limit (Jy) }\end{array}$} \\
\hline & & & $v=1$ & $v=2$ & & & & $\bar{v}=1$ & $v=2$ \\
\hline W And & 294 & $\mathrm{AK} / 10$ & 15.7 & 16.6 & X Gem & 588 & $\mathrm{AK} / 10$ & 18.5 & 18.0 \\
\hline T Aqr & 294 & $\mathrm{AK} / 10$ & 22.9 & 22.7 & BU Gem & 294 & $\mathrm{AK} / 10$ & 18.1 & 15.9 \\
\hline $\mathrm{RV} \mathrm{Aqr}$ & 294 & $\mathrm{AK} / 10$ & 24.3 & 23.5 & $\eta \mathrm{Gem}$ & 294 & $\mathrm{AK} / 10$ & 18.7 & 16.0 \\
\hline$\chi \mathrm{Aqr}$ & 294 & $\mathrm{AK} / 10$ & 23.4 & 25.6 & $\mathrm{~S}$ Her & 294 & $\mathrm{AK} / 10$ & 14.7 & 15.8 \\
\hline W Aql & 588 & $\mathrm{AK} / 10$ & 16.5 & 16.8 & S Her & 233 & FISPE & 11.1 & 11.1 \\
\hline RS Aql & 588 & $\mathrm{AK} / 10$ & 18.5 & 16.7 & T Her & 588 & $\mathrm{AK} / 10$ & 13.3 & 13.1 \\
\hline T Ari & 294 & $\mathrm{AK} / 10$ & 14.4 & 16.2 & W Her & 294 & $\mathrm{AK} / 10$ & 18.9 & 17.9 \\
\hline R Aur & 294 & $\mathrm{AK} / 10$ & 19.3 & 19.3 & W Her & 233 & FISPE & 7.0 & 7.0 \\
\hline S Aur & 294 & $\mathrm{AK} / 10$ & 17.6 & 16.4 & X Her & 117 & FISPE & 9.5 & 9.5 \\
\hline U Aur & 294 & $\mathrm{AK} / 10$ & 15.2 & 14.8 & RS Her & 588 & $\mathrm{AK} / 10$ & 12.2 & 11.6 \\
\hline RU Aur & 294 & $\mathrm{AK} / 10$ & 18.1 & 17.7 & UV Her & 294 & $\mathrm{AK} / 10$ & 15.7 & 16.0 \\
\hline TU Aur & 294 & $\mathrm{AK} / 10$ & 15.9 & 12.9 & UW Her & 233 & FISPE & 17.1 & 17.1 \\
\hline UU Aur & 298 & $\mathrm{AK} / 10$ & 15.6 & 19.1 & $\mathrm{~g} \mathrm{Her}$ & 233 & FISPE & 5.4 & 5.4 \\
\hline UZ Aur & 233 & FISPE & 6.6 & 6.6 & S Hya & 294 & $\mathrm{AK} / 10$ & 19.7 & 20.8 \\
\hline R Boo & 588 & $\mathrm{AK} / 10$ & 12.0 & 12.3 & T Hya & 294 & $\mathrm{AK} / 10$ & 24.7 & 24.7 \\
\hline S Boo & 588 & $\mathrm{AK} / 10$ & 13.9 & 15.6 & U Hya & 294 & $\mathrm{AK} / 10$ & 24.3 & 22.3 \\
\hline V Boo & 294 & $\mathrm{AK} / 10$ & 21.7 & 19.7 & V Hya & 294 & $\mathrm{AK} / 10$ & 28.6 & 28.6 \\
\hline W Boo & 235 & $\mathrm{AK} / 10$ & 15.4 & 18.4 & RU Hya & 294 & $\mathrm{AK}^{\prime} / 10$ & 47.3 & 47.3 \\
\hline RV Boo & 294 & $\mathrm{AK} / 10$ & 17.9 & 22.1 & S Lac & 294 & $\mathrm{AK} / 10$ & 15.9 & 18.6 \\
\hline RW Boo & 294 & $\mathrm{AK} / 10$ & 16.1 & 16.7 & R Lyn & 294 & $\mathrm{AK} / 10$ & 15.6 & 15.6 \\
\hline VZ Cam & 294 & $\mathrm{AK} / 10$ & 14.5 & 12.2 & R Lyr & 280 & FISPE & 5.1 & 5.1 \\
\hline V Cnc & 588 & $\mathrm{AK} / 10$ & 18.2 & 18.4 & W Lyr & 294 & $\mathrm{AK} / 10$ & 21.1 & 18.2 \\
\hline W Cnc & 588 & $\mathrm{AK} / 10$ & 16.3 & 15.9 & V Mon & 294 & $\mathrm{AK} / 10$ & 22.1 & 21.8 \\
\hline X Cnc & 294 & $\mathrm{AK} / 10$ & 13.7 & 13.3 & X Mon & 294 & $\mathrm{AK} / 10$ & 19.8 & 17.7 \\
\hline RS Cnc & 294 & $\mathrm{AK} / 10$ & 12.0 & 9.3 & SY Mon & 294 & $\mathrm{AK} / 10$ & 19.3 & 20.2 \\
\hline R CVn & 294 & $\mathrm{AK} / 10$ & 15.7 & 15.6 & CL Mon & 294 & $\mathrm{AK} / 10$ & 26.9 & 28.0 \\
\hline V CVn & 294 & $\mathrm{AK} / 10$ & 11.7 & 9.1 & $\mathrm{R}$ Oph & 294 & $\mathrm{AK} / 10$ & 36.4 & 36.4 \\
\hline Y CVn & 294 & $\mathrm{AK} / 10$ & 11.7 & 13.0 & $\mathrm{X}$ Oph & 294 & $\mathrm{AK} / 10$ & 24.5 & 26.1 \\
\hline $\mathrm{S} \mathrm{CMi}$ & 294 & $\mathrm{AK} / 10$ & 22.8 & 26.0 & $\mathrm{Z} \mathrm{Oph}$ & 294 & $\mathrm{AK} / 10$ & 20.0 & 22.8 \\
\hline V CMi & 294 & $\mathrm{AK} / 10$ & 15.6 & 18.9 & RT Oph & 294 & $\mathrm{AK} / 10$ & 19.8 & 19.3 \\
\hline S Cas & 588 & $\mathrm{AK} / 10$ & 12.8 & 13.3 & XOri & 294 & $\mathrm{AK} / 10$ & 23.6 & 24.1 \\
\hline U Cas & 588 & $\mathrm{AK} / 10$ & 14.4 & 15.2 & $\alpha$ Ori & 588 & $\mathrm{AK} / 10$ & 32.3 & - \\
\hline V Cas & 588 & $\mathrm{AK} / 10$ & 17.0 & 16.1 & S Peg & 588 & $\mathrm{AK} / 10$ & 15.3 & 16.8 \\
\hline SV Cas & 588 & $\mathrm{AK} / 10$ & 24.8 & 24.8 & $\mathrm{ZPeg}$ & 294 & $\mathrm{AK} / 10$ & 17.8 & 15.9 \\
\hline PZ Cas & .588 & $\mathrm{AK} / 10$ & 16.2 & 18.5 & UU Peg & 294 & $\mathrm{AK} / 10$ & 21.8 & 20.7 \\
\hline X Cep & 294 & $\mathrm{AK} / 10$ & 14.9 & 15.0 & $\mathrm{R}$ Per & 233 & FISPE & 9.6 & 9.6 \\
\hline$\mu$ Cep & 294 & $\mathrm{AK} / 10$ & 17.0 & 17.0 & U Per & 588 & $\mathrm{AK} / 10$ & 16.4 & 17.6 \\
\hline R Cet & 294 & $\mathrm{AK} / 10$ & 27.3 & 23.6 & RR Per & 233 & FISPE & 11.7 & 11.7 \\
\hline R Com & 588 & $\mathrm{AK} / 10$ & 11.4 & 12.2 & SU Per & 233 & FISPE & 7.2 & 7.2 \\
\hline R CrB & 59 & $\mathrm{AK} / 10$ & 61.0 & 52.0 & GI Per & 294 & $\mathrm{AK} / 10$ & 14.5 & 16.0 \\
\hline V CrB & 233 & FISPE & 8.1 & 8.1 & VZ Sge & 294 & $\mathrm{AK} / 10$ & 11.6 & 12.9 \\
\hline RR CrB & 233 & FISPE & 5.4 & 5.4 & RR Sco & 353 & $\mathrm{AK} / 10$ & 52.3 & 56.6 \\
\hline $\mathrm{RS} \mathrm{CrB}$ & 233 & FISPE & 5.6 & 5.6 & BG Ser & 294 & $\mathrm{AK} / 10$ & 18.7 & 20.0 \\
\hline P Cyg & 294 & $\mathrm{AK} / 10$ & 12.9 & 12.9 & $\mathrm{ZTau}$ & 294 & $\mathrm{AK} / 10$ & 20.2 & 18.4 \\
\hline R Cyg & 233 & FISPE & 5.1 & 5.1 & RX Tau & 294 & $\mathrm{AK} / 10$ & 18.1 & 18.9 \\
\hline R Cyg & 204 & $\mathrm{AK} / 10$ & 15.8 & 14.6 & CE Tau & 233 & FISPE & 11.3 & 11.3 \\
\hline U Cyg & 294 & $\mathrm{AK} / 10$ & 11.9 & 12.8 & R Tri & 147 & $\mathrm{AK} / 10$ & 19.9 & 20.6 \\
\hline V Cyg & 294 & $\mathrm{AK} / 10$ & 12.4 & 12.7 & R UMa & 294 & $\mathrm{AK} / 10$ & 15.4 & 15.4 \\
\hline W Cyg & 294 & $\mathrm{AK} / 10$ & 11.3 & 11.9 & S UMa & 588 & $\mathrm{AK} / 10$ & 16.8 & 17.2 \\
\hline Z Cyg & 294 & $\mathrm{AK} / 10$ & 11.9 & 12.8 & T UMa & 588 & $\mathrm{AK} / 10$ & 11.5 & 11.6 \\
\hline RT Cyg & 294 & $\mathrm{AK} / 10$ & 15.4 & 14.1 & Y UMa & 353 & $\mathrm{AK} / 10$ & 15.1 & 15.1 \\
\hline RU Cyg & 294 & $\mathrm{AK} / 10$ & 12.6 & 11.5 & Z UMa & 294 & $\mathrm{AK} / 10$ & 10.8 & 13.5 \\
\hline RV Cyg & 294 & $\mathrm{AK} / 10$ & 12.0 & 12.9 & ST UMa & 294 & $\mathrm{AK} / 10$ & 13.4 & 14.1 \\
\hline SX Cyg & 294 & $\mathrm{AK} / 10$ & 20.6 & 17.8 & S UMi & 294 & $\mathrm{AK} / 10$ & 19.2 & 18.5 \\
\hline TT Cyg & 294 & $\mathrm{AK} / 10$ & 12.0 & 12.4 & U UMi & 588 & $\mathrm{AK} / 10$ & 14.6 & 14.0 \\
\hline AF Cyg & 233 & FISPE & 6.3 & 6.3 & RR UMi & 294 & $\mathrm{AK} / 10$ & 15.6 & 15.6 \\
\hline CN Cyg & 294 & $\mathrm{AK} / 10$ & 14.3 & 16.0 & R Vir & 588 & $\mathrm{AK} / 10$ & 14.7 & 14.4 \\
\hline V1057 Cyg & 294 & $\mathrm{AK} / 10$ & 16.2 & 15.1 & S Vir & 294 & $\mathrm{AK} / 10$ & 22.9 & 23.9 \\
\hline $\mathrm{R}$ Del & 294 & $\mathrm{AK} / 10$ & 18.2 & 19.3 & U Vir & 294 & $\mathrm{AK} / 10$ & 20.7 & 21.7 \\
\hline U Del & 294 & $\mathrm{AK} / 10$ & 16.9 & 16.9 & RX Vul & 294 & $\mathrm{AK} / 10$ & 17.6 & 19.2 \\
\hline V Del & 588 & $\mathrm{AK} / 10$ & 11.6 & 12.9 & IRC -10502 & 294 & $\mathrm{AK} / 10$ & 23.3 & 26.1 \\
\hline R Dra & 294 & $\mathrm{AK} / 10$ & 21.9 & 22.1 & IRC +00357 & 294 & $\mathrm{AK} / 10$ & 26.7 & 23.7 \\
\hline T Dra & 294 & $\mathrm{AK} / 10$ & 33.7 & 26.3 & IRC +00402 & 294 & $\mathrm{AK} / 10$ & 24.0 & 27.8 \\
\hline RY Dra & 294 & $\mathrm{AK} / 10$ & 13.2 & 13.2 & IRC +10216 & 294 & $\mathrm{AK} / 10$ & 20.4 & 19.5 \\
\hline UX Dra & 233 & FISPE & 5.1 & 5.1 & $(=\mathrm{CW}$ Leo $)$ & & & & \\
\hline R Gem & 294 & $\mathrm{AK} / 10$ & 19.0 & 19.0 & & & & & \\
\hline
\end{tabular}

range. For the red giants, including NML Cyg, the emission is confined to a velocity spread of 7 to $15 \mathrm{~km} \mathrm{~s}^{-1}$. The supergiants VY CMa, S Per, and VX Sgr show emission over about $30 \mathrm{~km} \mathrm{~s}^{-1}$. One-half of these values $\left(3.5\right.$ to $15 \mathrm{~km} \mathrm{~s}^{-1}$ ) are reasonable velocities to associate with mass loss from late-type stars. The large range of 
TABLE III. $v=3$ results.

\begin{tabular}{lccc}
\hline \hline \multicolumn{1}{c}{ Star } & Spectrometer & $\begin{array}{c}\text { Peak flux } \\
\text { density } \\
(\mathrm{Jy})\end{array}$ & $\begin{array}{c}\text { Radial } \\
\text { velocity } \\
\left(\mathrm{km} \mathrm{s}^{-1}\right)\end{array}$ \\
\hline RX Boo & AK/10 & $<22$ & \\
VY CMa & AK/10 & $110 \pm 22$ & 41 \\
R Cas & FISPE & $<1.0$ & \\
NML Cyg & AK/10 & $<17$ & \\
NML Cyg & FISPE & $<1.5$ & \\
X Cyg & AK/10 & $<8$ & \\
X Cyg & FISPE & $<1.7$ & \\
U Her & FISPE & $<1.0$ & \\
W Hya & AK/10 & $<12$ & \\
R Leo & AK/10 & $<26$ & \\
R LMi & AK/10 & $<11$ & \\
VX Sgr & AK/10 & $70 \pm 14$ & 4 \\
RT Vir & AK/10 & $<22$ & \\
IRC+10216 & AK $/ 10$ & $<18$ & \\
\hline \hline
\end{tabular}

velocities covered by the FISPE observations (up to 140 $\mathrm{km} \mathrm{s}^{-1}$ ) would have been sufficient to detect emission similar to the high-velocity emission of water masers in some regions of star formation (Walker et al. 1977). At some velocities, not necessarily related to the stellar velocity, to the velocity of the optical emission, or to optical absorption, strong, narrow, $\mathrm{SiO}$ emission is observed. In the supergiants such as VY CMa or S Per there are more than two strong narrow peaks. If the $v=1$ and $v=2$ lines originated in the expanding stellar envelopes, there should be a correlation between the total width of the line profile and the period of the light variations (Dickinson and Chaisson 1973; Dickinson et al. 1978a). No such correlation was found, which indicates that these masers are situated close to the stars themselves.

The line profiles of the spectra range from simple to complex. With our higher sensitivity and resolution, the complexity of the spectra has increased relative to earlier observations. Earlier descriptions of $o$ Cet and other stars as having double-peaked emission similar to $\mathrm{OH}$ masers are oversimplified. SiO maser spectra consist of many blended features that are frequently unresolved with the $0.2-\mathrm{km} \mathrm{s}^{-1}$ resolution of some of the spectra in Figs. 1-8. Typical blended features appear to be 1 to $2 \mathrm{~km} \mathrm{~s}^{-1}$ wide. In most sources only a few velocities are favored, and they dominate the spectra. The VLBI observations confirm this conclusion (Moran et al. 1979; Lane et al. 1980).

\section{b) Weak Satellite Emission}

Snyder et al. (1978) discovered a weak broad-line emission associated with the dominant narrow masers that had been previously studied. They referred to this emission as a "pedestal," and felt that the center velocity of the pedestal yields stellar radial velocities: The "pedestal represents a useful extension to the radial velocity method introduced by Reid and Dickinson (1976)."

We find that weak satellite maser emission (i.e., broad, blended emission often over a wide velocity range) is common in our larger sample of stars. This satellite maser emission is what Snyder et al. (1978) observed, but with their lower resolution and sensitivity the masers appeared to merge together into a broad feature. We cannot separate these weak satellites from the stronger lines as clearly as Snyder et al. (1978) have done. At the time of our observations there was no narrow emission for many stars and in most stars the strong emission lines blended into the weak satellite masers. It is often difficult to separate weak masers in the satellite region from stronger ones closer in with the high resolution and sensitivity of these spectra.

Snyder et al. (1978) removed the main-line emission, then fit a parabola to the quasi-thermal pedestal to obtain a stellar velocity. If we were to attempt this process on the present epoch spectrum of VY CMa, the center velocity would be in the range of 18 to $20 \mathrm{~km} \mathrm{~s}^{-1}$ instead of the $13.1 \pm 0.1 \mathrm{~km} \mathrm{~s}^{-1}$ found by Snyder et al. (1978). The weaker system near $40 \mathrm{~km} \mathrm{~s}^{-1}$, which includes a narrow maser feature and weak satellite masers, would indicate a second long-period variable star, in the analysis of Snyder et al. (1978), which is unlikely. Furthermore, $v=1$ and $v=2$ spectra for many of the stars with clear satellite emission yield different stellar velocities. The spectra of Figs. 1-8 indicate that weak maser emission can occur within a limited velocity range, that more masers (or stronger masers) tend to be at the central velocities, but that there is no obvious parabolic or Gaussian distribution of emission. In particular, the stars previously found to have pedestals, VY CMa, R Cas, $o$ Cet, NML Cyg, W Hya, R Leo, VX Sgr, and IK Tau, appear not to have simple parabolic pedestals, but to have a blend of weak masers adjacent to the strong emission.

\section{c) Variability}

The observations between 9 and 17 May 1979 show significant variations in stars that were observed repeatedly, usually for pointing corrections. The data were too sparse, however, to distinguish between temporal intensity variations and polarization effects, owing to the differing position angle of the $E$ vector during the observations. While intensity variations in these sources are well known on longer time scales, the $\mathrm{SiO}$ masers also are known to be strongly polarized in the $J=2-1$ transition (Troland et al. 1979) and to have some linear polarization at $J=1-0$ (Schwartz et al. 1977). The cause of the present variations is therefore uncertain.

Table II contains further evidence of the intensity variability of SiO masers. W And, R Aur, and S Vir would have been detected at 5,10 , and 8 times the noise level, respectively, if they were as strong as their previously reported flux density at the epoch of these observations (Blair and Dickinson 1977; Dickinson et al. 1978b; Lépine, Le Squeren, and Scalise 1978). A fourth known star not detected, RR Sco, was at low elevations and the $3 \sigma$ limit of $\sim 55 \mathrm{Jy}$ is close to the previously re- 

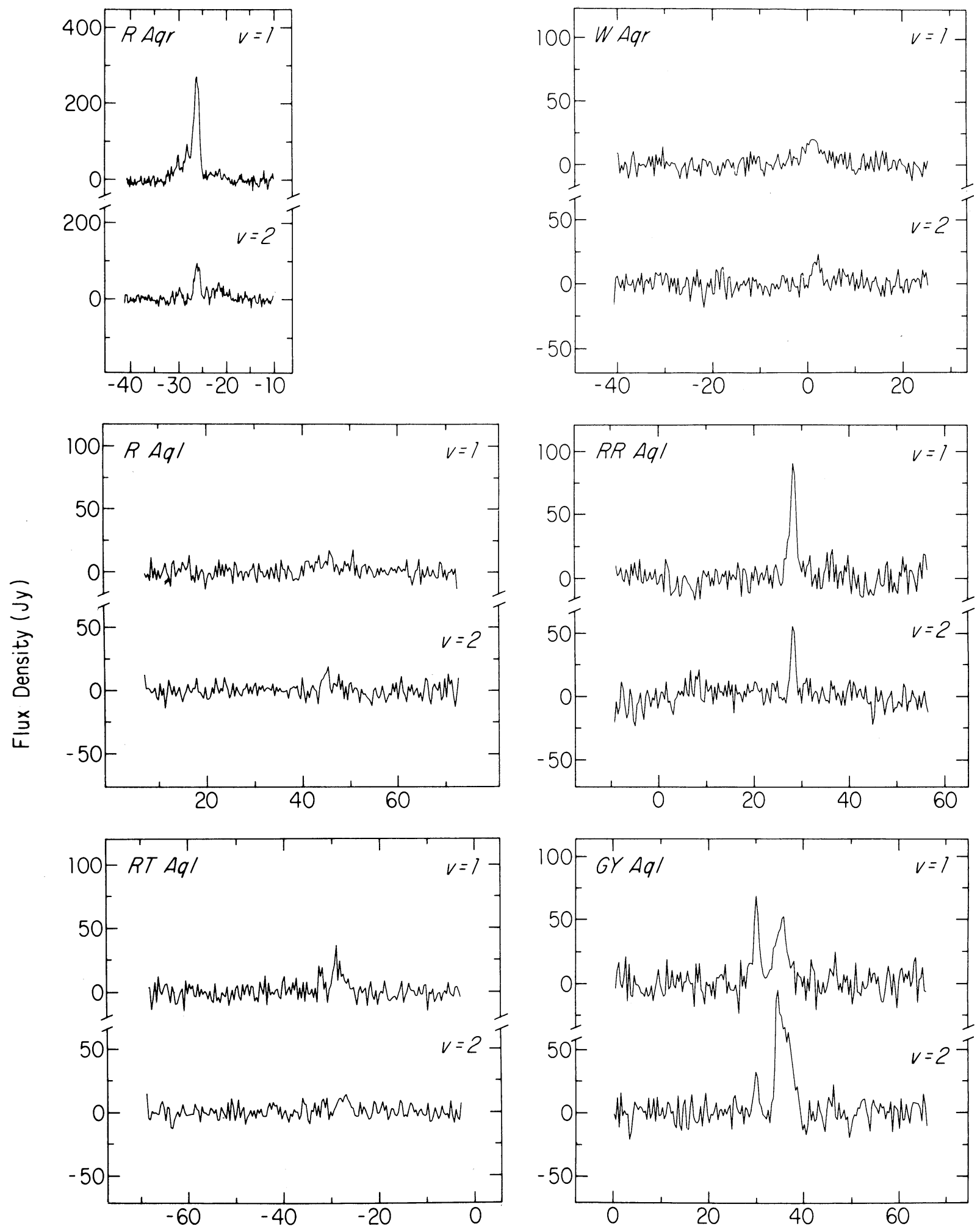

Radial Velocity $\left(\mathrm{km} \mathrm{s}^{-1}\right)$

FIG. 1. $J=1-0, v=1$, and $v=2 \mathrm{SiO}$ line profiles detected in the present work. Unless noted the spectrometer was either the $\mathrm{AK} / 10$ or $\mathrm{AK} / 5$ with uniform weighting. The two transitions are presented on the same flux density scale for each star. The radial velocities are relative to the local standard of rest. 

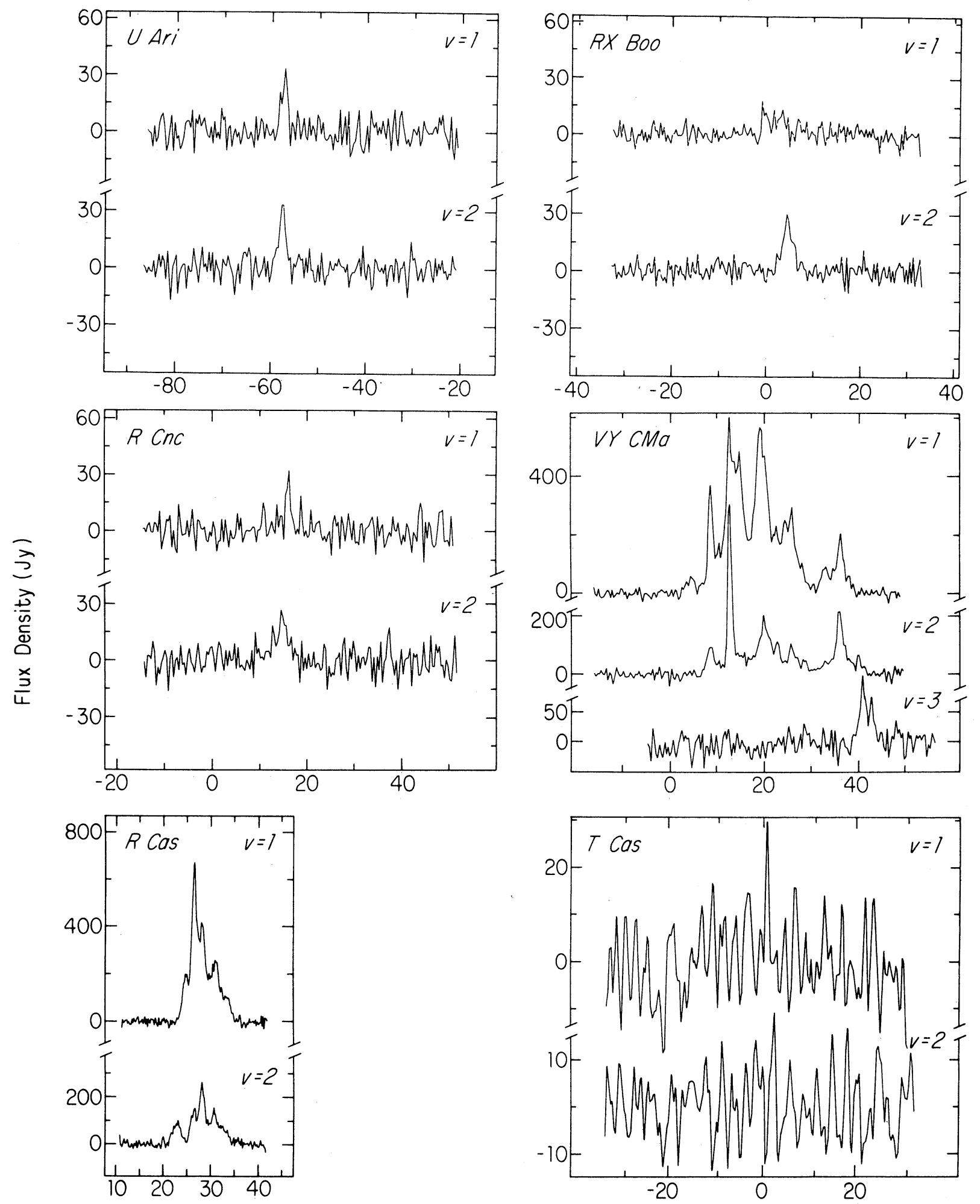

Radial Velocity $\left(\mathrm{km} \mathrm{s}^{-1}\right)$

FIG. 2. Same as Fig. 1. Where $v=3$ was detected (VY CMa) the flux density scale has been enlarged. The spectra for T Cas have been smoothed with Hanning weighting. 


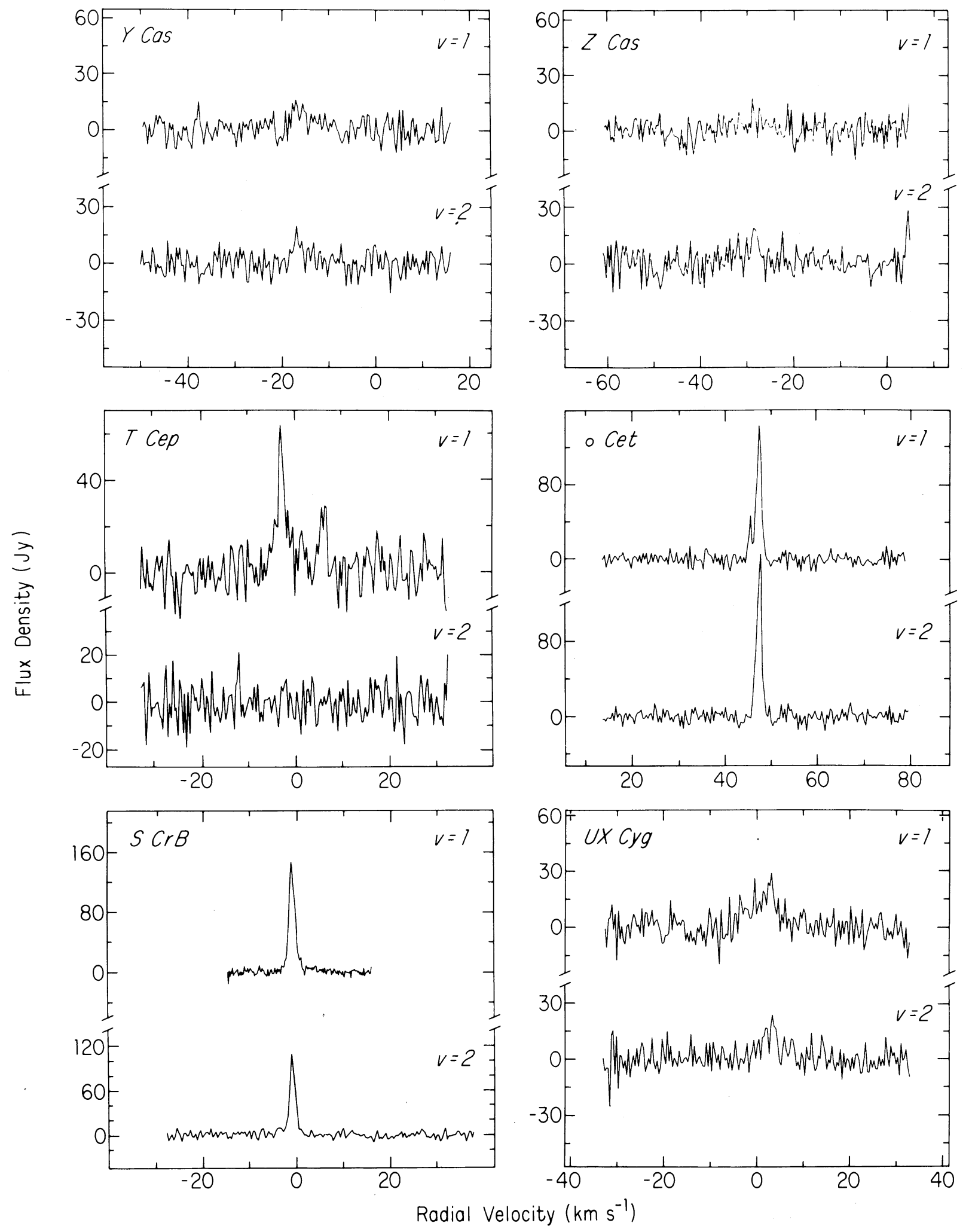

FIG. 3. Same as Fig. 1. 

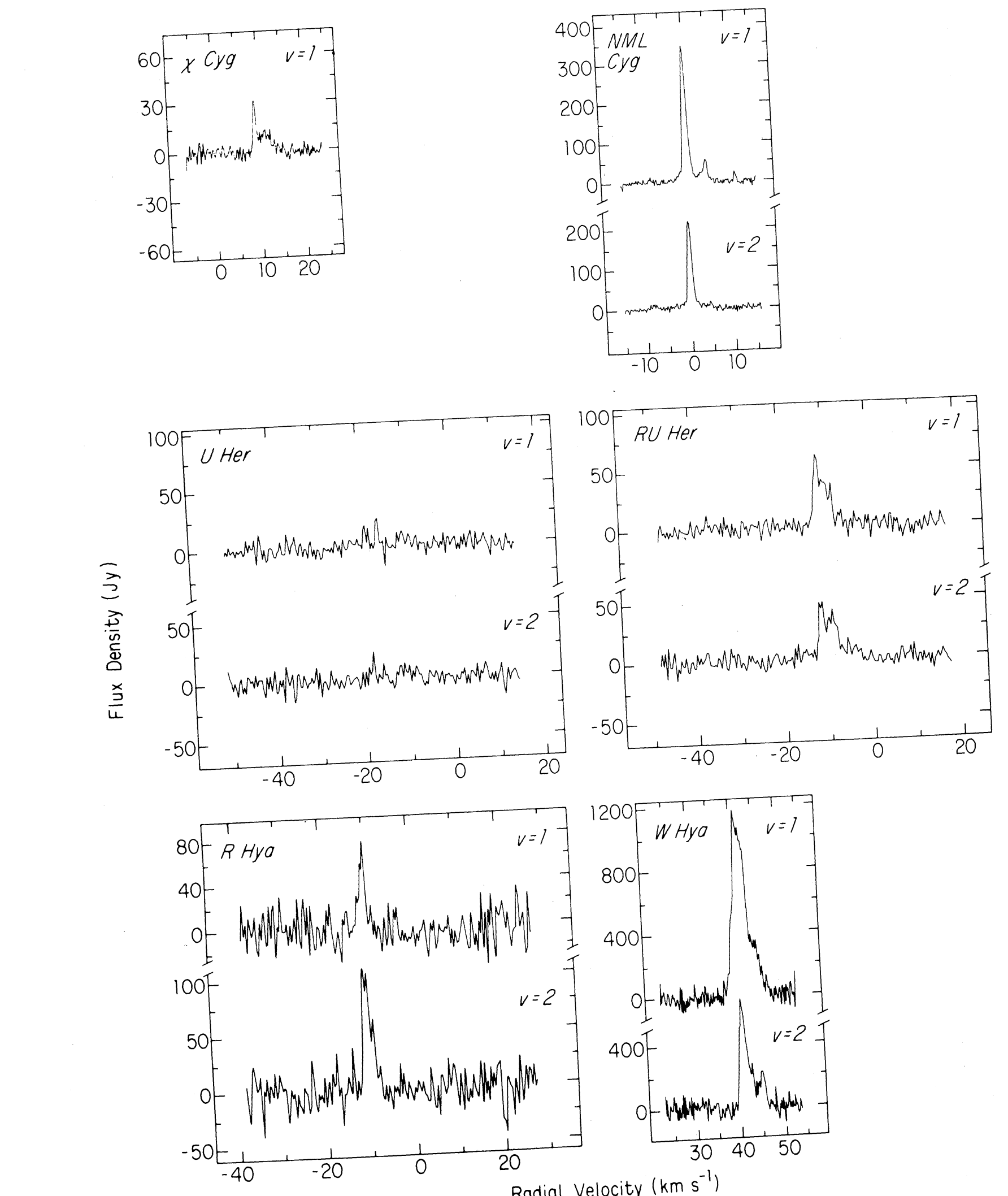

FIG. 4. Same as Fig. 1. 

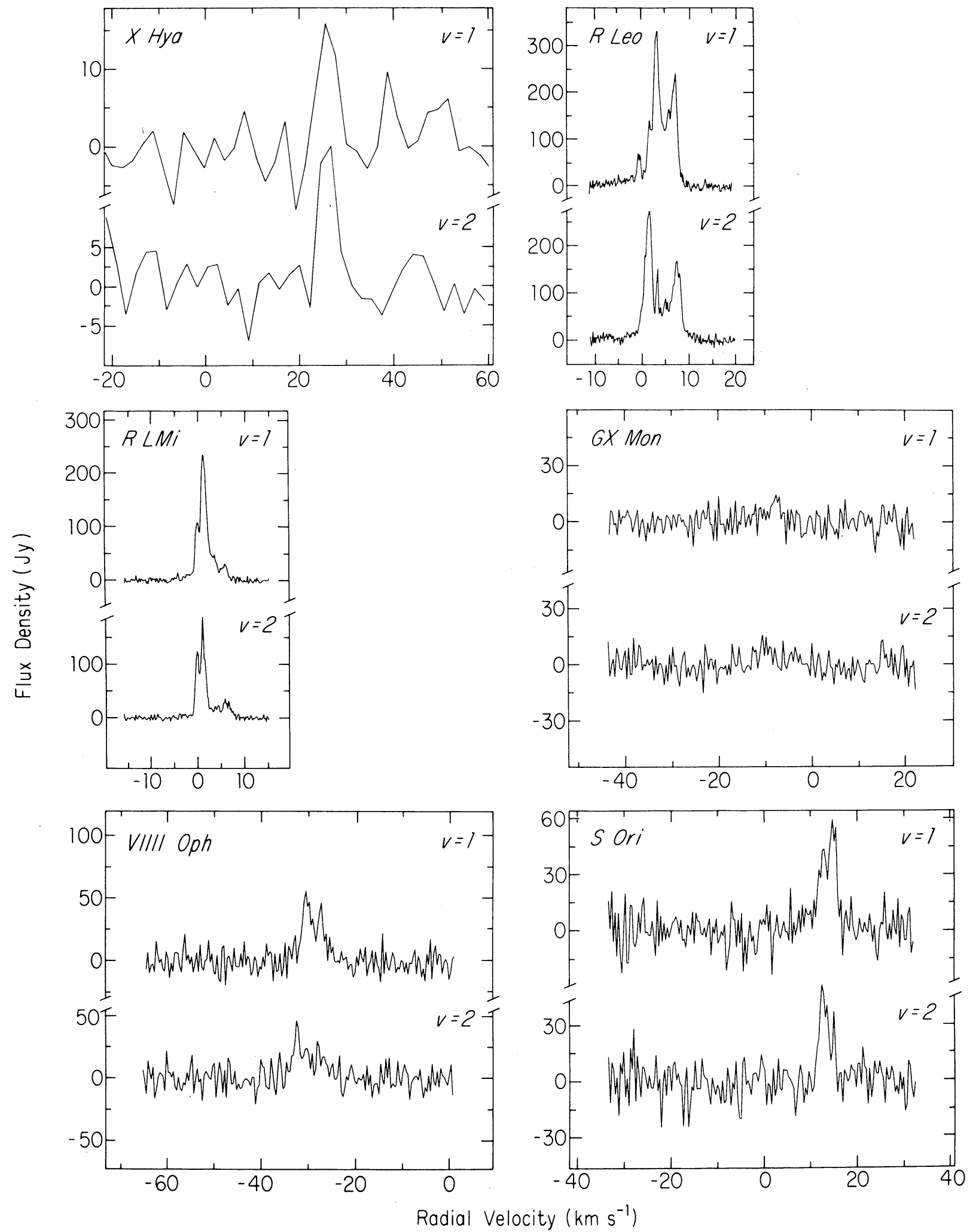

FIG. 5. Same as Fig. 1. The spectra for X Hya were taken with the FISPE spectrometer. 

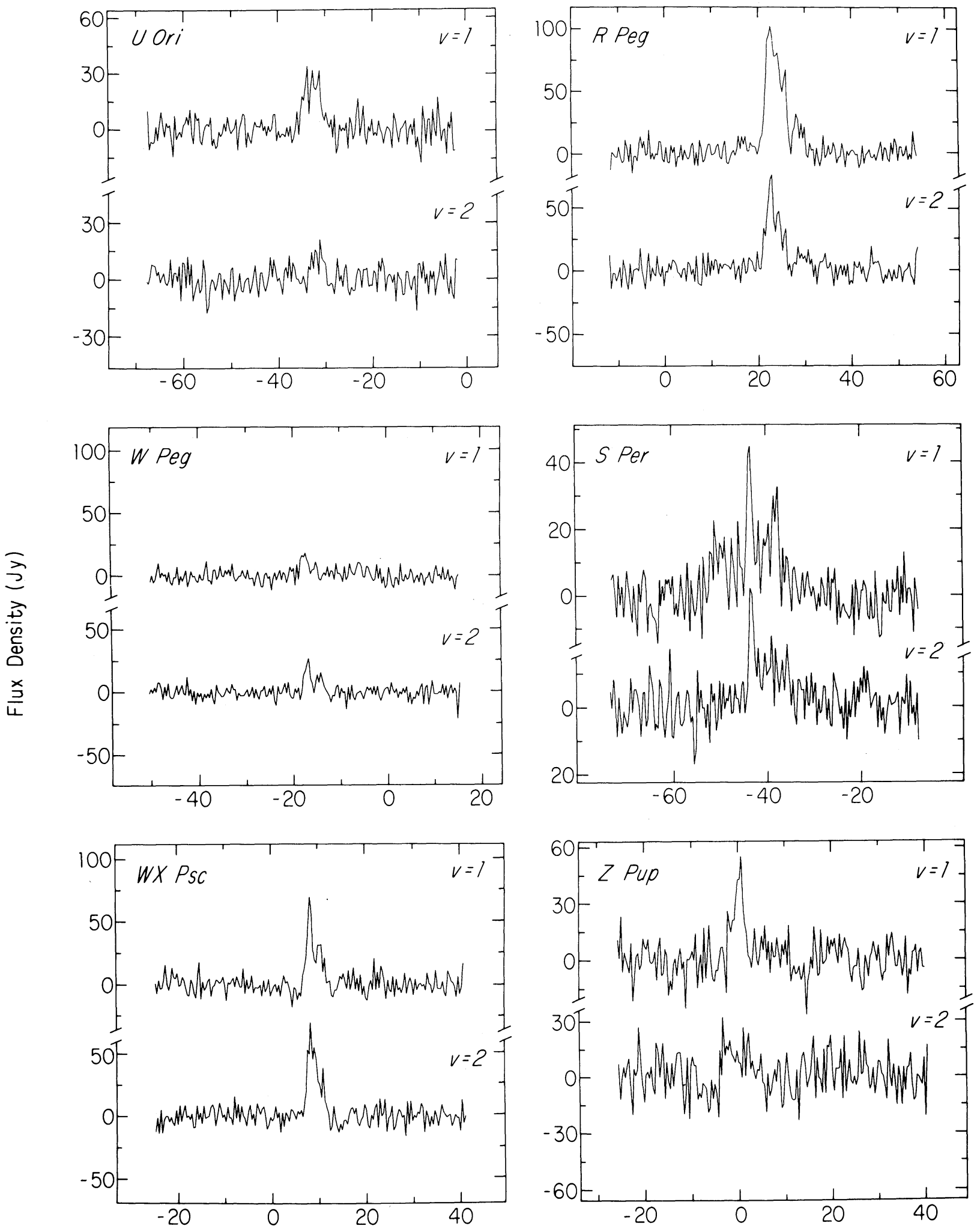

Rodial Velocity $\left(\mathrm{km} \mathrm{s}^{-1}\right)$

FIG. 6. Same as Fig. 1. 

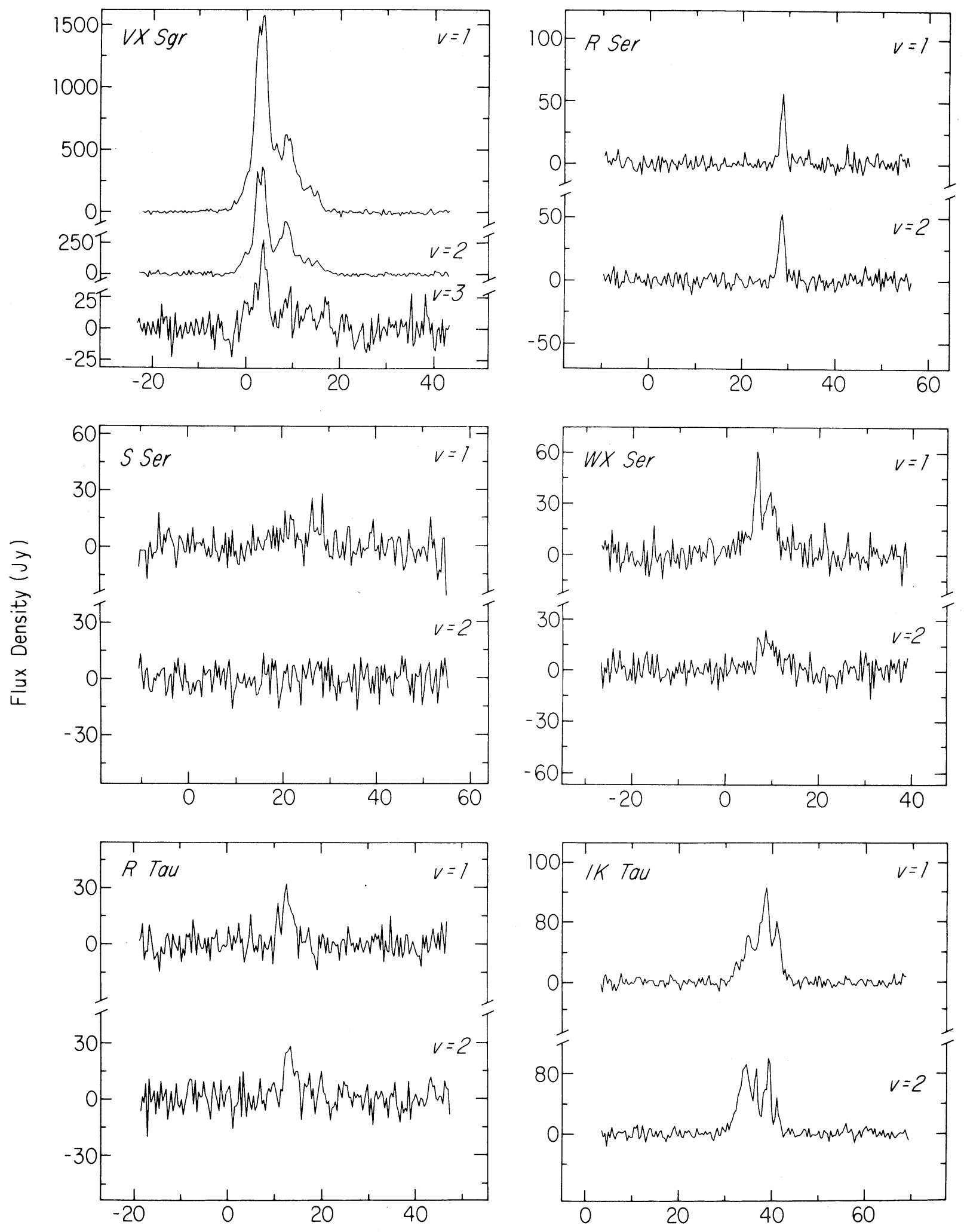

Radial Velocity $\left(\mathrm{km} \mathrm{s}^{-1}\right)$

FIG. 7. Same as Fig. 1. Where $v=3$ was detected (VX Sgr) the flux density scale has been enlarged. 

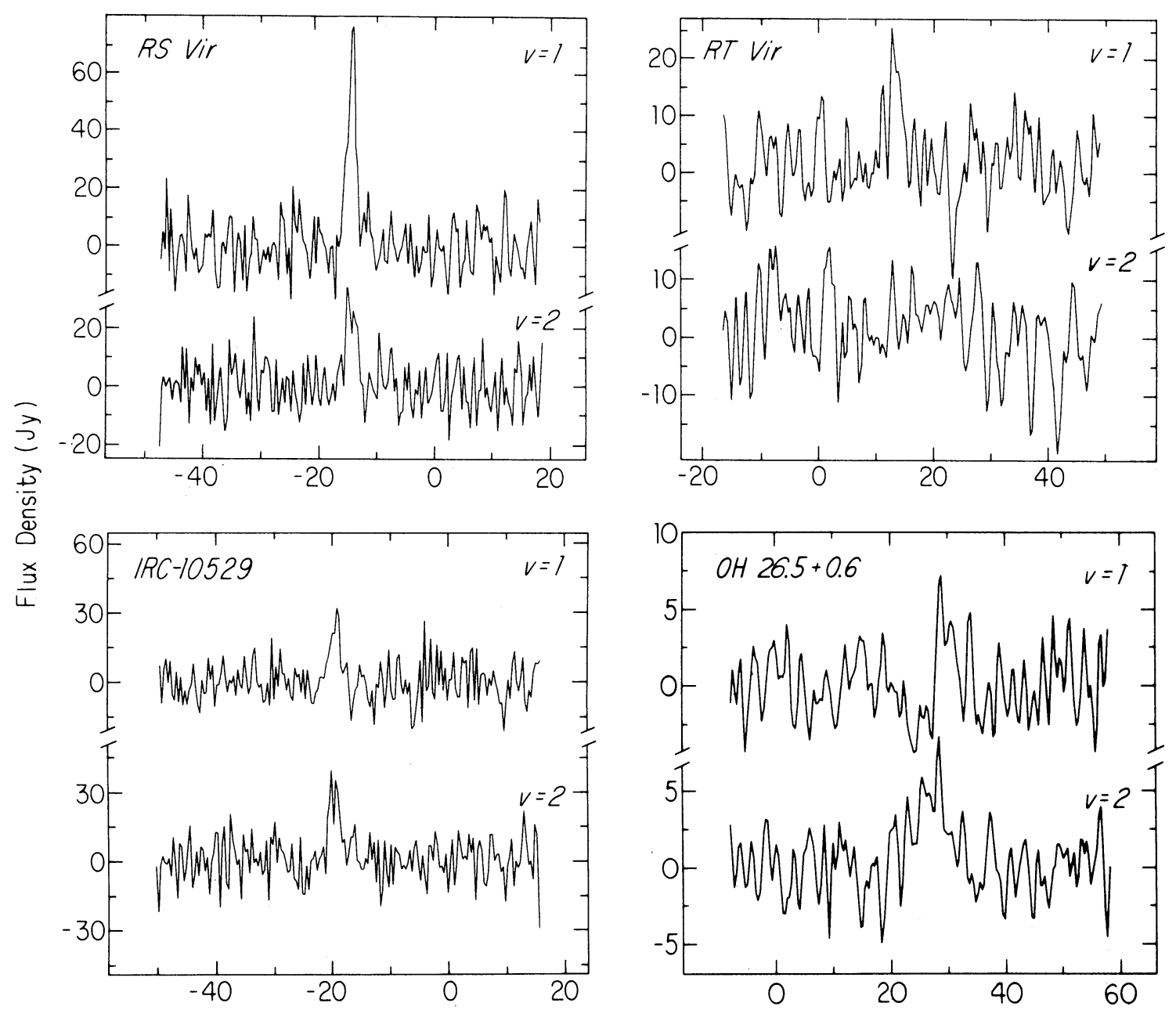

Radial Velocity $\left(\mathrm{km} \mathrm{s}^{-1}\right)$

FIG. 8. Same as Fig. 1. The spectra for RT Vir and $\mathrm{OH} 26.5+0.6$ have been smoothed with Hanning weighting.

ported $J=2-1$ flux (Dickinson et al. 1978b). The $J=$ 1-0 line has never been reported.

\section{d) Sample Completeness}

There are several methods that can be used to estimate the completeness of the sample. In a homogeneous, spherical distribution of maser stars, the $\log N-\log I$ relationship (Fig. 9) would have a slope of -1.5 , where $N$ is the total number of stars with an intensity greater than I. Clearly the total sample marked by the crosses in Fig. 9 is heterogeneous, possibly the cause of the complex shape of the curve. A more homogeneous sample, that of the 34 M-type Miras marked by footnote "a" in Table I, shows an underabundance below an intensity of $10^{-18.5}$ $\mathrm{W} \mathrm{m}^{-2}$ owing to an incompleteness in the sample of masers. This flux corresponds to a distance limit of about $300 \mathrm{pc}$. Since the observations attempted to be complete to a greater distance, it appears these results are sensitivity limited. If the distribution of masers is not spherical, but confined to a thin disk, the slope of the $\log N$ $\log I$ relation would be -1 . The late-type Miras in our sample have a scale height above the galactic plane of 165-300 pc (Smak 1966). Therefore we do not expect this to strongly affect our $\log N-\log I$ relation given the characteristic distance of completeness estimated above.

A second test for completeness is the luminosityvolume test (von Hoerner 1974). In this test one calculates the volume $V$ of the sphere with a radius equal to the actual distance to the star divided by the volume $V_{m}$, corresponding to the maximum distance at which this particular star could have been detected. If the space distribution were uniform (and spherical), the frequency distribution of this function would be constant with a mean value of 0.5 . 


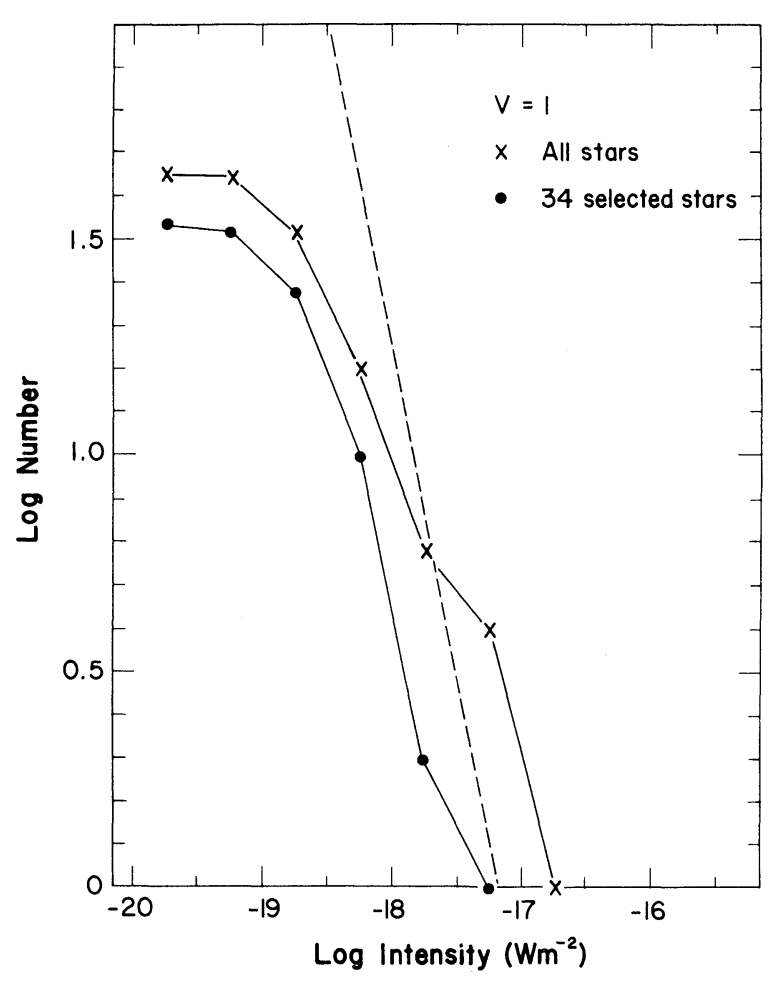

FIG. 9. The $\log N-\log I$ relation. The curve marked with x's includes all the stars in Table I. The curve marked with closed circles includes the 34 stars marked by a footnote "a" in Table 1 . The dashed line has a slope of -1.5 , which one expects for a homogeneous, spherical distribution.

The quantity of interest, $V / V_{m}$, is equal to the signal-to-noise ratio to the -1.5 power and is independent of the assumed stellar distance. It is therefore strongly dependent on the detection criteria. We have calculated $V / V_{m}$ using both flux density and integrated intensity separately. The resulting distributions are significantly weighted toward low values and exclude a mean value of 0.5. This supports the conclusion of the $\log N-\log I$ test that we are mainly dealing with nearby sources.

We made a third test for completeness, by projecting each detected star onto the galactic plane and counting the number of stars in heliocentric regions of equal areas. The radii were generated with the function

$$
r_{n}^{2}=r_{n-1}^{2}+r_{1}^{2}
$$

where we used $r_{1}=200 \mathrm{pc}$. The results are presented in Table IV. If the stars were in a disk with uniform space density, then there would be an equal number in each interval. Once again we see problems in the distribution beyond a radius of $\sim 300 \mathrm{pc}$.

If the noise in the spectra for all M-type Miras observed (positive and negative detections) is corrected to the stellar distance to get a peak luminosity limit, we get the distribution shown in Fig. 10. Peak luminosity is the luminosity per hertz that corresponds to the observed peak flux density. The numbers above each column show
Distribution of Upper Limits to Peak Luminosities

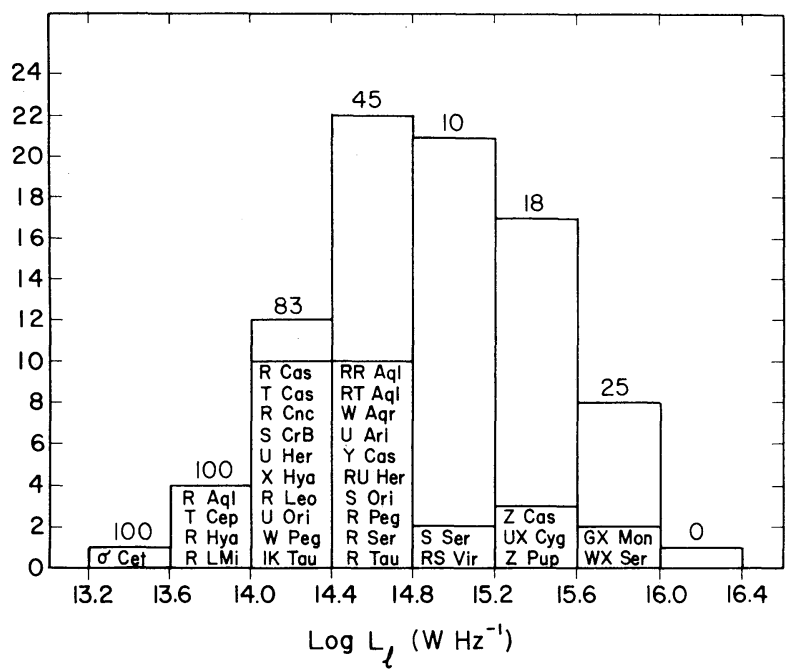

FIG. 10. The peak luminosity limits for all stars, both detected and undetected. The peak noise luminosity is the noise in an observed spectrum corrected to the stellar distance (see text).

the detection percentage. The negative results are predominantly at higher peak luminosity limits, again indicating that successful detections occurred for the closer stars. If the noise were to be reduced by an order of magnitude, through longer integration for instance, this figure predicts that the number of detected sources would double, but still there would be a number of negative results because of sensitivity limitations.

\section{e) Luminosity Function}

We would like to estimate the luminosity function of $\mathrm{SiO}$ masers. The luminosity distribution of the stars marked by footnote "a" in Table I is presented in Fig. 11. The mean luminosity based on Fig. 11 is $3.6 \times 10^{20}$ $\mathrm{W}$. The true luminosity function is expected to have more weak masers, decreasing this mean value.

We attempted to correct the observed luminosity distribution for incompleteness by using the upper limits for the undetected stars. This technique is applied on the peak luminosities and peak luminosity limits because the

TABLE IV. Equal surface test.

\begin{tabular}{cc}
\hline $\begin{array}{c}\text { Radius from } \\
\text { the Earth } \\
(\mathrm{pc})\end{array}$ & $\begin{array}{c}\text { Number of SiO } \\
\text { maser stars } \\
\text { in each area }\end{array}$ \\
$0-200$ & 7 \\
$200-283$ & 8 \\
$283-346$ & 2 \\
$346-400$ & 5 \\
$400-447$ & 2 \\
$447-490$ & 1 \\
$490-529$ & 2 \\
$529-566$ & 0 \\
$566-600$ & 1 \\
\hline \hline
\end{tabular}




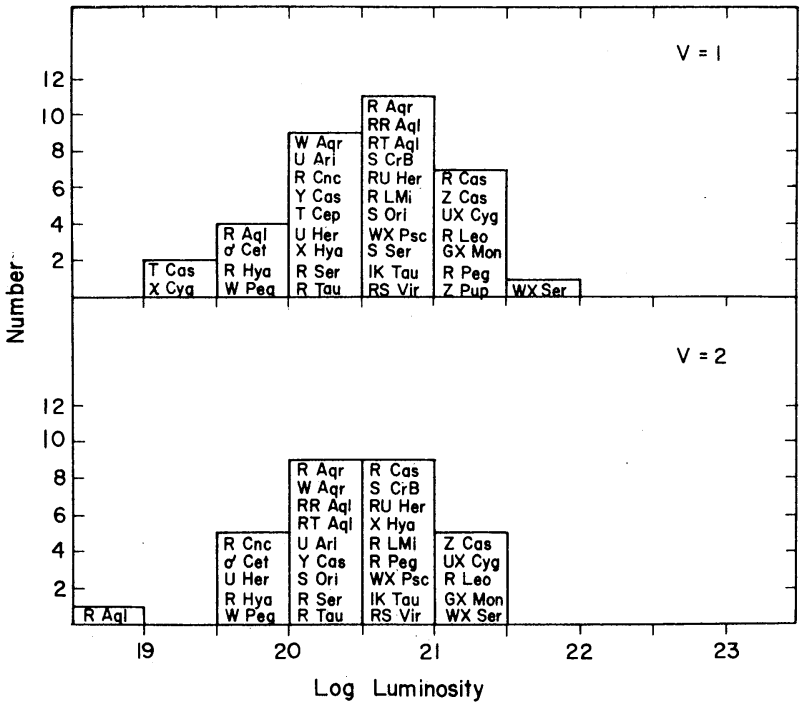

FIG. 11. The luminosity distribution for the stars with footnote "a" in Table $\mathrm{I}$.

detection criteria were dominated by flux density deviations. Once a peak luminosity $\left(\mathrm{W} \mathrm{Hz}^{-1}\right)$ is found, it can be converted to integrated luminosity by using linewidths.

Our method differs from that recently described by Avni et al. (1980). We use information of three types: detected luminosities, upper limits for nondetections, and equivalent noise limits for the detections. Their method uses only the first two types of information and requires slightly more mathematics. However, when we applied their technique to our data, the resultant luminosity functions were identical except for minor differences at low luminosities $(14 \lesssim \log L \lesssim 14.2)$, where there is not much information.

Our procedure is first to convert the flux density limits for all objects (detected and undetected) to peak luminosity limits (the distribution shown in Fig. 10). Likewise, the peak flux densities of the detected objects are converted to peak luminosities. For every value of luminosity the stars with a luminosity limit less than or equal to that value are examined. We then calculate the fraction of stars with a peak luminosity greater than or equal to the test value, with respect to the total number of stars examined. These fractions are a function of luminosity. The complement is the cumulative luminosity distribution. The differential peak luminosity function is shown in Fig. 12, smoothed to 0.3 in $\log \left(\mathrm{W} \mathrm{Hz}^{-1}\right)$. The more luminous portion $\left(\log L_{p}>15\right)$ of the function is well defined, as confirmed by the observed luminosity distribution (the histogram in Fig. 12). For $\log L_{p} \lesssim 14.4$, large deviations from a smooth function would be expected because of the lack of information we have about masers with extremely low luminosity. While it is impossible to restore the luminosity function at low luminosities with the present sample, we believe that it is highly improbable that a significant fraction of Miras have $\log L_{p}<14$. A power-law fit yields the relation $N$ $\sim L_{p}^{-0.9}$ for $14.5<\log L_{p}<16$. This is half the slope found for $\mathrm{OH}$ maser emission from Type II OH/IR sources by Baud et al. (1980).

We are trying to find the $\mathrm{SiO}$ luminosity function and would like to describe its characteristics, not only over the range where it can be restored accurately, but also outside that range. At the high-luminosity end, we know that none of the $\sim 85$ stars has a $\log L_{p}$ larger than 15.8. The fitted power law, in contrast, has a fairly strong high-luminosity wing because of its low exponent. The power-law description must break down fairly soon above $\log L_{p} \sim 16$. Something similar happens at the low-luminosity side. We found $15 \mathrm{SiO}$ emitters among

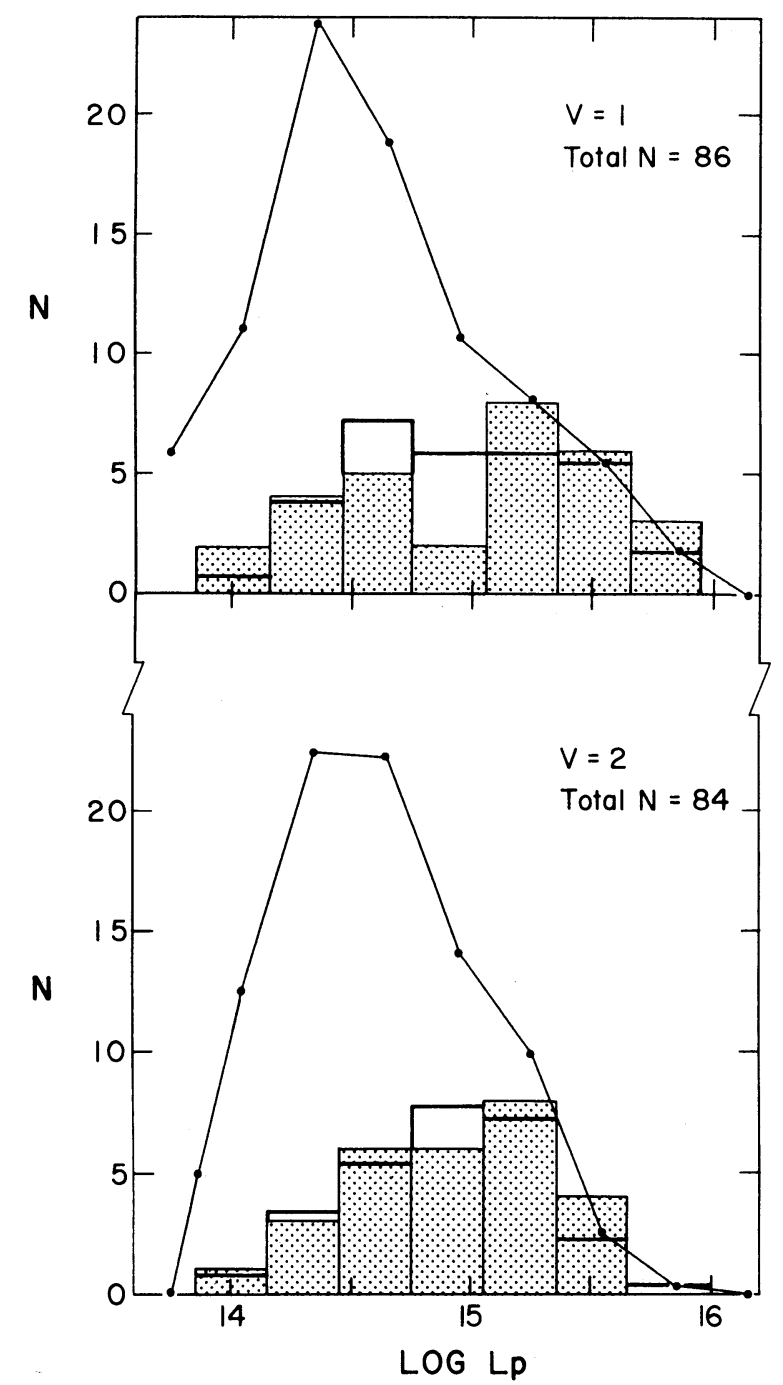

FIG. 12. The luminosity function for $\mathrm{SiO}$ masers found in Mira variables. This function is based on the observed distribution (shaded) and corrected for incompleteness, as described in the text. The predicted observed distribution is the bold histogram. (Upper) $v=1$ luminosity function; (lower) $v=2$ luminosity function. 


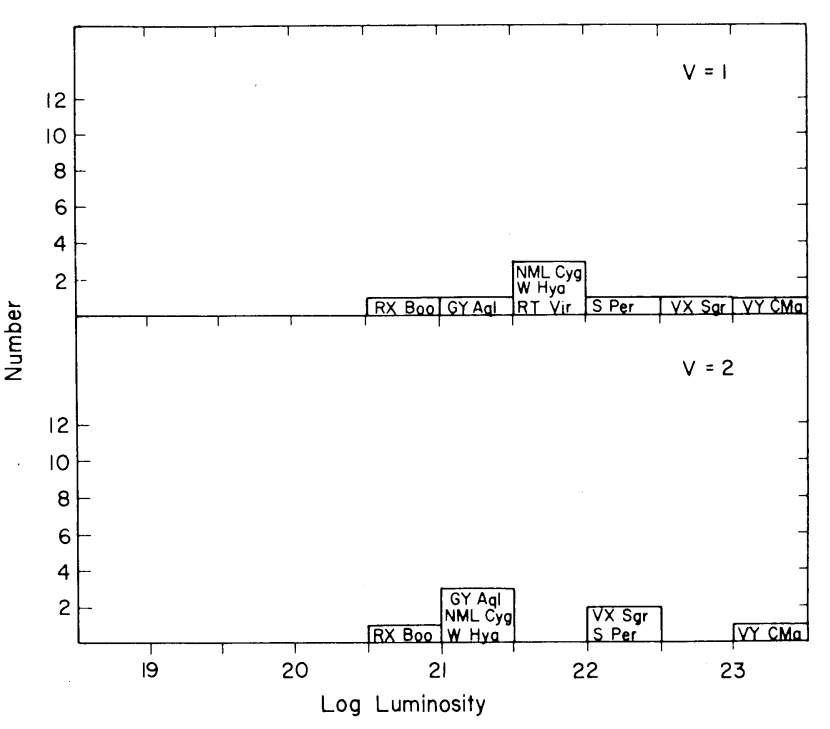

FIG. 13. The luminosity distribution for supergiant and semiregular stars listed in Table I.

the 17 stars with the lowest detection limits. That makes it highly improbable that a significant fraction of M-type Mira variables have $\log L_{p}$ below 14. Any power-law description of the luminosity function must break down below $\log L_{p}=14.5$. Thus while $N \sim L_{p}^{-0.7}$ describes the luminosity function adequately over an important range, it is completely wrong outside that range. The $\mathrm{SiO}$ luminosity function (mean $\log L_{p} \sim 14.6$ ) is fairly narrow and extends over only two orders of magnitude.

The observed luminosity distribution of the masers associated with the supergiant and semiregular stars is shown in Fig. 13. As a class these masers are among the more luminous stellar masers. Even if the closer distance to VY CMa of 400 pc (Hyland et al. 1969) were adopted, it would still be the most luminous, with a luminosity of $1.8 \times 10^{22} \mathrm{~W}$. The $\mathrm{OH}$ and $\mathrm{H}_{2} \mathrm{O}$ masers associated with this star are unusual for stellar masers. The individual $\mathrm{H}_{2} \mathrm{O}$ masers in VY CMa are more compact, of smaller spot size, and more luminous than those in Mira variables (compare Rosen et al. 1978 with Spencer et al. 1979). The same is true for the $\mathrm{OH}$ masers (compare Reid and Muhleman 1978 with Bowers et al. 1980).

\section{f) Correlations Among SiO Luminosity, Period, and Bolometric Luminosity}

The $v=1$ and $v=2 \mathrm{SiO}$ luminosities were plotted versus stellar period, and no correlation was found. The scatter was fairly uniform over 300 to 450 days. For periods longer than 450 days, there seemed to be a deficiency of low-luminosity $\mathrm{SiO}$ masers, probably due to the method of selecting and observing these rarer stars.

The mean luminosity of the observed masers corre-

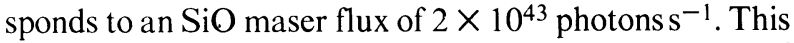
flux is about ten times the $\mathrm{H}_{2} \mathrm{O}$ emission photon rate of about $2 \times 10^{42}$ photons $^{-1}$ for these same stars. For Mira variables the number of photons at $4 \mu \mathrm{m}$ available to pump the observed masers is about $10^{44}$ photons $\mathrm{s}^{-1}$. If the $\mathrm{SiO}$ masers are radiatively pumped, it is reasonable to expect a correlation between $\mathrm{SiO}$ luminosity and stellar luminosity. Cahn and Elitzur (1979) found a correlation between $\mathrm{SiO}$ luminosity and the stellar luminosity at $4 \mu \mathrm{m}$. In the larger sample of masers presently available, there is no correlation between $\mathrm{SiO}$ luminosity and the total bolometric luminosity as derived from Cahn and Wyatt (1978). If we limit the stars to those in common with Cahn and Elitzur (1979), there is no correlation with $4 \mu \mathrm{m}$, contrary to their conclusion.

\section{g) The Ratio of the $v=1$ and $v=2$ Line Intensities}

A quantity which is distance independent is the ratio $Q$ of the $v=1$ to the $v=2$ integrated intensities. The distribution of $\log Q$ is plotted in Fig. 14. For a Gaussian distribution, there is less than a $5 \%$ probability from the student's $t$ test that the mean would be displaced from zero by chance. Thus we conclude that on the average the $v=1$ line is $1.4 \pm 0.1$ times stronger than the $v=2$ line in the same star.

This result may be compared with the average value $\bar{Q}=0.95 \pm 0.50$ obtained for a sample of 13 stars and Orion in 1977 by Schwartz, Bologna, and Waak (1979). The two results are, within the errors, compatible.

If, according to Elitzur (1980), the $\mathrm{SiO}$ masers are in the stellar atmosphere and collisionally pumped, one would expect a correlation between $Q$ and the effective temperature of the star because (again according to Elitzur) the densities in the atmospheres are about the same for all Miras. We find no such correlation nor any between $Q$ and luminosity $(v=1)$ or $Q$ and period.

\section{h) The $v=3$ Emission}

Following the announcement of detection of $v=3$ emission in W Hya and VX Sgr by Scalise and Lépine

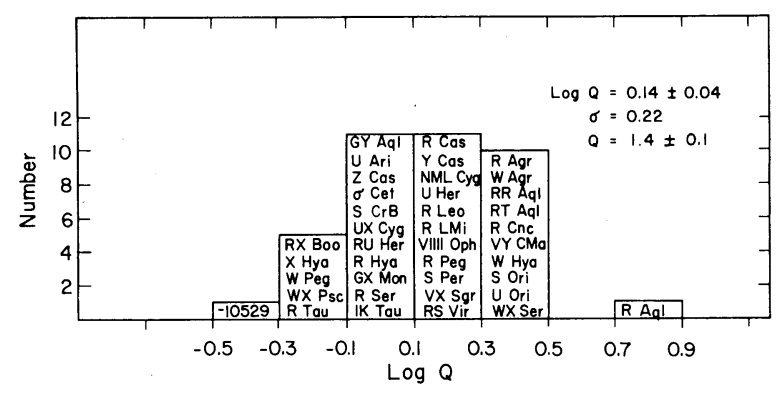

FIG. 14. The ratio of the $v=1$ to the $v=2$ integrated intensity $(Q)$ for the stars with footnote " $\mathrm{a}$ " in Table I. The offset of $\log Q$ corresponds to a ratio of $1.4 \pm 0.1$. 
(1978), we expected to confirm their sources and find new ones because we had a more sensitive system. As shown in Table III, this was not the case. We unexpectedly measured a $3 \sigma \mathrm{rms}$ upper limit in W Hya of $12 \mathrm{Jy}$, about one-third of the peak flux previously reported. This is much lower than the upper limit set by Buhl et al. (1974). Scalise and Lépine suggest that $v=3$ emission is related to the light curve of the star. Our observational results neither support nor refute this hypothesis.

\section{CONCLUSIONS}

These observations, taken nearly simultaneously in the $v=1, v=2$, and $v=3$ lines, greatly increase our data base for study of $\mathrm{SiO}$ maser emission. The high resolution and high sensitivity of these spectra show no evidence for "quasi-thermal pedestals" of emission, even though weak satellite masers are quite common. The main features are clearly blends of weak masers. There is a close velocity coincidence between line components in the $v=1$ and $v=2$ spectra of the same star. Only the velocity of $v=3$ in VY CMa is highly anomalous. The $\mathrm{SiO}$ maser sample is now complete to approximately 300 pc, limited by sensitivity. We have detected 13 new sources. A luminosity function corrected for sample incompleteness has been derived and can be described as a power law with an exponent of -0.7 . The luminosity functions are the same for $v=1$ and $v=2$, regardless of their true shapes. The $v=1$ line is on the average 1.4 times stronger than the $v=2$ line in the same star. No global properties of the stars correlate with the $\mathrm{SiO}$ luminosity, indicating that the masers are close to the stars, where local properties may be important. Yet the stellar temperature does not correlate with $\mathrm{SiO}$ luminosity. This complicates the situation and further understanding may require simultaneous observations at optical and infrared wavelengths and at 43 - or $86-\mathrm{GHz} \mathrm{SiO}$ masers.

The authors would like to thank the many people at MPIfR who helped in these observations, especially the engineering and observing staffs. Our colleague V. Pankonin participated in the observations reported here and in their initial analysis. H.E.M. is grateful to the MPIfR for providing travel funds. We thank J. H. Cahn for providing distances to many of the Mira variables and S. von Hoerner for helpful discussions. A.W. was a guest of the National Radio Astronomy Observatory during the analysis of these data.

\section{REFERENCES}

Allen, C. W. (1973). Astrophysical Quantities, 3rd ed. (Athlone, London).

Altenhoff, W. J., Baars, J. W. M., Downes, D., Pankonin, V., Wink, J. E., Winnberg, A., Schwartz, P. R., Spencer, J. H., Matthews, H. E., Genzel, R., and Olnon, F. M. (1980). Astron. J. 85, 9.

Andersson, C., Johansson, L. E. B., Goss, W. M., Winnberg, A., and Nguyen-Q-Rieu (1974). Astron. Astrophys. 30, 475.

Avni, Y., Soltan, A., Tannanbaum, H., and Zamorani, G. (1980). Astrophys. J. 238, 800.

Balister, M., Batchelor, R. A., Haynes, R. F., Knowles, S. H., McCulloch, M. G., Robinson, B. J., Wellington, K. J., and Yabsley, D. C. (1977). Mon. Not. R. Astron. Soc. 180, 415.

Baud, B., Habing, H. J., Matthews, H. E., and Winnberg, A. (1980). Astron. Astrophys. (in press).

Blair, G. N., and Dickinson, D. F. (1977). Astrophys. J. 215, 552.

Bowers, P. F., Reid, M. J., Johnston, K. J., Spencer, J. H., and Moran, J. M. (1980). Astrophys. J. 242 (in press).

Buhl, D., Snyder, L. E., Lovas, F. J., and Johnson, D. R. (1974). Astrophys. J. Lett. 192, L97.

Cahn, J. H. (1980). Private communication.

Cahn, J. H., and Elitzur, M. (1979). Astrophys. J. 231, 124.

Cahn, J. H., and Wyatt, S. P. (1978). Astrophys. J. 221, 163.

Dickinson, D. F., and Chaisson, E. J. (1973). Astrophys. J. Lett. 181, L135.

Dickinson, D. F., Reid, M. J., Morris, M., and Redman, R. (1978a). Astrophys. Lett. 220, L113.

Dickinson, D. F., Snyder, L. E., Brown, L. W., and Buhl, D. (1978b). Astron. J. 83, 36.

Elitzur, M. (1980). Astrophys. J. 240, 553.

Engels, D. (1979). Astron. Astrophys. Suppl. 36, 337.

Foy, R., Heck, A., and Mennessier, M. O. (1975). Astron. Astrophys. 43, 175.
Genzel, R., Downes, D., Schwartz, P. R., Spencer, J. H., Pankonin, V., and Baars, J. W. M. (1980). Astrophys. J. 239, 519.

Harvey, P. M., Bechis, K. P., Wilson, W. J., and Ball, J. A. (1974). Astrophys. J. Suppl. 27, 331.

Herbig, G. H. (1969). Mem. Soc. R. Sci. Liege, Ser. 8, 13.

Hjalmarson, A., and Olofsson, H. (1979). Astrophys. J. Lett. 234, L199.

Hyland, A. R., Becklin, E. E., Frogel, J. A., and Neugebauer, G. (1972). Astron. Astrophys. 16, 204

Hyland, A. R., Becklin, E. E., Neugebauer, G., and Wallerstein, G. (1969). Astrophys. J. 158, 619.

Kaifu, N., Buhl, D., and Snyder, L. E. (1975). Astrophys. J. 195, 359.

Keenan, P. C., Garrison, R. F., and Deutsch, A. J. (1974). Astrophys. J. Suppl. 28, 271.

Kleinmann, S. G., Dickinson, D. F., and Sargent, D. G. (1978). Astron. J. 83, 1206.

Kukarkin, B. V., Kholopov, P. N., Efremov, Yu. N., Kukarkin, N. P., Kurochkin, N. E., Medvedeva, G. I., Perova, N. B., Fedorovich, V. P., and Frolov, M. S. (1969-1976). General Catalog of Variable Stars, 3rd ed. and supplements (Astronomical Council, Academy of Sciences, Moscow).

Lane, A. P., Ho, P. T. P., Predmore, C. R., Moran, J. M., Genzel, R., Hansen, S. S., and Reid, M. J. (1980). In Interstellar Molecules, IAU Symposium No. 87, edited by B. Andrew (Reidel, Dordrecht).

Lépine, J. R. D., Le Squeren, A. M., and Scalise, E., Jr. (1978). Astrophys. J. 225, 869.

Low, F. J., Kurtz, R. F., Vrba, F. J., and Rieke, G. H. (1976). Astrophys. J. Lett. 206, L153.

Moran, J. M., Ball, J. A., Predmore, C. R., Lane, A. P., Huguenin, G. R., Reid, M. J., and Hansen, S. S. (1979). Astrophys. J. Lett. 231, L67. 
Neugebauer, G., and Leighton, R. B. (1969). Two-Micron Sky Survey, NASA SP-3047 (U.S. GPO, Washington, D.C.).

Olnon, F. M., Winnberg, A., Matthews, H. E., and Shultz, G. V. (1980). Astron. Astrophys. Suppl. 42, 119.

Reid, M. J., and Muhleman, D. O. (1978). Astrophys. J. 220, 229.

Rosen, B. R., Moran, J. M., Reid, M. J., Walker, R. C., Burke, B. F., Johnston, K. J., and Spencer, J. H. (1978). Astrophys. J. 222, 132.

Scalise, E., Jr., and Lépine, J. R. D. (1978). Astron. Astrophys. 65, L7.

Schwartz, P. R., Bologna, J. M., and Waak, J. A. (1979). Astron. J. 84, 1349.

Schwartz, P. R., Waak, J. A., Bologna, J. M., and Spencer, J. H. (1977). Bull. Am. Astron. Soc. 9, 354.

Smak, J. I. (1966). Annu. Rev. Astron. Astrophys. 4, 19.

Snyder, L. E., and Buhl, D. (1975). Astrophys. J. 197, 329.
Snyder, L. E., Dickinson, D. F., Brown, L. W., and Buhl, D. (1978). Astrophys. J. 224, 512.

Spencer, J. H., Johnston, K. J., Moran, J. M., Reid, M. J., and Walker, R. C. (1979). Astrophys. J. 230, 449.

Spencer, J. H., and Schwartz, P. R. (1975). Astrophys. J. Lett. 199, L111.

Spencer, J. H., Schwartz, P. R., Waak, J. A., and Bologna, J. M. (1977). Astron. J. 82, 706.

Troland, T. H., Heiles, C., Johnson, D. R., and Clark, F. O. (1979). Astrophys. J. 232, 143.

von Hoerner, S. (1974). In Galactic and Extragalactic Radio Astronomy, edited by G. L. Verschuur and K. I. Kellermann (Springer, New York), p. 353.

Walker, R. C., Johnston, K. J., Burke, B. F., and Spencer, J. H. (1977). Astrophys. J. Lett. 211, L135. 